\title{
Vibrational Analysis of Peptides, Polypeptides, and Proteins. V. Normal Vibrations of $\beta$-Turns
}

\author{
S. KRIMM and JAGDEESH BANDEKAR, Biophysics Research \\ Division and Department of Physics, The University of Michigan, Ann \\ Arbor, Michigan 48109
}

\begin{abstract}
Synopsis
The normal modes have been calculated for $\beta$-turns of types I, II, III, I', II', and III'. The complete set of frequencies is given for the first three structures; only the amide I, II, and III modes are given for the latter three structures. Calculations have been done for structures with standard dihedral angles, as well as for structures whose dihedral angles differ from these by amounts found in protein structures. The force field was that refined in our previous work on polypeptides. Transition dipole coupling was included, and is crucial to predicting frequency splittings in the amide I and amide II modes. The results show that in the amide I region, $\beta$-turn frequencies can overlap with those of the $\alpha$-helix and $\beta$-sheet structures, and therefore caution must be exercised in the interpretation of protein bands in this region. The amide III modes of $\beta$-turns are predicted at significantly higher frequencies than those of $\alpha$-helix and $\beta$-sheet structures, and this region therefore provides the best possibility of identifying $\beta$-turn structures. Amide $\mathrm{V}$ frequencies of $\beta$-turns may also be distinctive for such structures.
\end{abstract}

\section{INTRODUCTION}

The region in which a polypeptide chain reverses its direction by $\sim 180^{\circ}$ is often referred to as a $\beta$-turn. Such regions, consisting of the three peptide groups at the turn, comprise a significant component of globular proteins: in a recent analysis ${ }^{1}$ we found that in 38 nonhomologous proteins, $29 \%$ of the amino acids occur in $\beta$-turns.

Specific conformations for such $\beta$-turns were first characterized in detail by Venkatachalam ${ }^{2}$ on the basis of stereochemical criteria, although analogous structures were also proposed at this time from a study of the "cross- $\beta$ " conformation of fibrous proteins. ${ }^{3}$ Three main types of $\beta$-turns were described, ${ }^{2}$ all having an internal hydrogen bond between the $\mathrm{CO}$ group of residue 1 and the $\mathrm{NH}$ group of residue 4. Types I and II are nonhelical conformations, whereas type III corresponds to one turn of a 310 -helix. The standard form of the type II turn requires a glycine residue at the 3-position, there being no such restrictions for the other turns. Structures which have mirror-image arrangements of the backbone atoms were also found to be possible, ${ }^{2}$ and are designated as types $\mathrm{I}^{\prime}, \mathrm{II}^{\prime}$, and III'.

A subsequent analysis of eight protein structures ${ }^{4}$ revealed that five additional types of turns could exist, designated IV, V, V', VI, and VII. 
Type IV comprises any of the $\beta$-turns of types I III' in which the values of two or more angles $\phi$ and $\psi$ at residues 2 and 3 differ by at least $40^{\circ}$ from the typical angles for these types. A type $\mathrm{V}$ turn corresponds to a sevenmembered hydrogen-bonded ring conformation (compared to a $10-\mathrm{mem}$ bered ring for the others). The type VI turn has a cis proline at the 3position. In the type VII turn the distance between the $\mathrm{C}^{\alpha}$ atoms of residues 1 and 4 is less than $7 \AA$, a minimal criterion required of all $\beta$-turns, but instead of a reversal in direction there is a "kink" in the chain. A later detailed analysis of 29 protein structures ${ }^{5}$ revealed that all of the above types of $\beta$-turns occur: type $I$ is the most frequent ( $42 \%$ of the total of 421 observed), followed by types III (18\%), II (15\%), and IV (8\%). Mirror-image types are less frequent: type $\mathrm{I}^{\prime}$ had a frequency of $3 \%$, type $\mathrm{II}^{\prime}$ a frequency of about $5 \%$, and type III' a frequency of $3 \%$. The other types were found rarely. It should be noted that in this study, ${ }^{5} \beta$-turns were considered "ideal" if any of the $\phi$ and $\psi$ at residues 2 and 3 did not differ by more than $50^{\circ}$ from the values typical for that type. With this definition not even all ideal $\beta$-turns had an internal hydrogen bond, whereas some nonideal turns did have such a bond.

In view of their significant contribution to the structure of proteins, it is important that physical methods be available to identify and characterize $\beta$-turns. Vibrational spectroscopic techniques, namely ir and Raman, can be useful for this purpose. Although experimental studies on model compounds ${ }^{6,7}$ can suggest the existence of such conformations, it is essential to be able to predict the vibrational frequencies of $\beta$-turns in order to have a sound theoretical basis for assigning characteristic bands to such structures. We have, therefore, calculated the normal vibration frequencies of several types of $\beta$-turns. A preliminary report of the results has been given $^{1}$; we present here the details of the calculations.

\section{NORMAL VIBRATION CALCULATIONS}

There are two main problems associated with the calculation of the normal modes of vibration of $\beta$-turns: the large variability in the angles $(\phi, \psi)_{2}$ and $(\phi, \psi)_{3}$ found for any particular type of turn, ${ }^{5}$ and the large variety of side-chain residues associated with these positions. ${ }^{5}$ We have chosen to deal with the first problem by computing the frequencies associated with $\beta$-turns having "standard" angles $2,4,5$ and then computing the effects on the frequencies of variations from the standard structures. In this way it is possible to develop some feeling for the importance that variations in different parts of the structure have in shifting the frequencies. We have not dealt with the second problem in any detail at this stage, using an alanyl residue for all side chains (except in type II, where a glycyl residue was used in the 3-position; variations with alanyl at this position will be discussed later). This is probably not too bad an approximation, since initially we will be most interested in the characteristic modes associated with the peptide group and with the backbone, and these are not likely to be strongly affected by the side-chain structure beyond the $\mathrm{C}^{\beta}$-position. 
The system chosen to represent a type I $\beta$-turn is $\mathrm{CH}_{3}-\mathrm{CO}-(\mathrm{Ala})_{4}$ $\mathrm{NH}-\mathrm{CH}_{3}$, and it is shown schematically in Fig. 1. An isolated $\beta$-turn can be adequately described by two sets of dihedral angles, namely $(\phi, \psi)_{2}$ and $(\phi, \psi)_{3}$, and it might be thought, therefore, that the model system $\mathrm{CH}_{3}$ $\mathrm{CO}-(\mathrm{Ala})_{2}-\mathrm{NH}-\mathrm{CH}_{3}$ would suffice to calculate the normal modes of $\beta$-turns in proteins. In fact, a calculation for such a structure showed that this was not the case. The $\beta$-turns in proteins have peptide groups adjoining the three of the tripeptide system, and transition dipole coupling ${ }^{8,9}$ between these groups is significantly different for the case of three as compared to five groups. We have therefore selected the larger model as more representative of $\beta$-turns in proteins. (Conversely, it should be kept in mind that the frequencies calculated here will not necessarily be applicable to small peptide $\beta$-turns having fewer than five peptide groups.)

The bond lengths and angles used in these calculations were the same as in our previous work. ${ }^{10-12}$ The dihedral angles at residues 1 and 4 were maintained, in the standard $\beta$-turn types I-III, at values consistent with the continuation of the chains in an antiparallel-chain pleated-sheet structure, viz., $(\phi, \psi)_{1}=(\phi, \psi)_{4}=-139^{\circ}, 135^{\circ}$. For $\beta$-turn types I'-III', these values of $\phi, \psi$ were sterically disallowed, and we therefore used $(\phi, \psi)_{1}=$ $(\phi, \psi)_{4}=-60^{\circ}, 120^{\circ}$. We computed the complete normal mode spectrum of $\beta$-turn types I-III (and their mirror-image conformations), and for these we took the typical dihedral angles ${ }^{2}$ as: $\mathrm{I}-(\phi, \psi)_{2}=-60^{\circ},-30^{\circ},(\phi, \psi)_{3}$ $=-90^{\circ}, 0^{\circ} ; \mathrm{II}-(\phi, \psi)_{2}=-60^{\circ}, 120^{\circ},(\phi, \psi)_{3}=80^{\circ}, 0^{\circ} ; \mathrm{III}-(\phi, \psi)_{2}=-60^{\circ}$, $-30^{\circ},(\phi, \psi)_{3}=-60^{\circ},-30^{\circ}$ (and the negative of these values for $I^{\prime}-I^{\prime} I^{\prime}$ ).

In the standard structures, internal hydrogen bonds were formed, viz.,

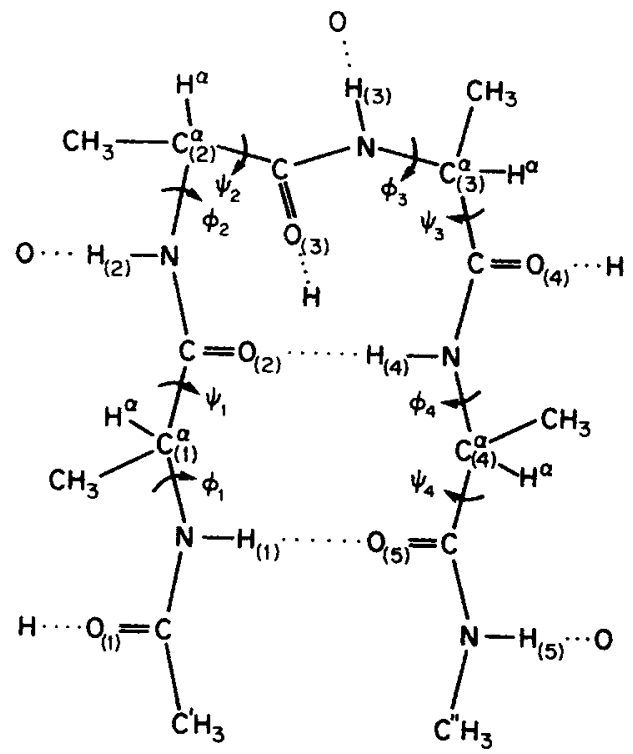

Fig. 1. Schematic illustration of a $\beta$-turn of $\mathrm{CH}_{3}-\mathrm{CO}-\left(\mathrm{A} \mathrm{l}_{-}\right)_{-}-\mathrm{NH}_{-} \mathrm{r}, \mathrm{H}_{3}$ with external hydrogen bonds included. 
$\mathrm{H}_{(1)} \ldots \mathrm{O}_{(5)}$ and $\mathrm{H}_{(4)} \cdots \mathrm{O}_{(2)}$. When structural variations were examined these distances changed, and in some cases, these bonds were no longer present. In order to take account of this effect, we allowed the hydrogen-bond force constant, $f(\mathrm{H} \ldots \mathrm{O})$, to decrease linearly from its value ${ }^{10}$ of $0.125 \mathrm{md} \AA^{-1}$ at $r(\mathrm{H} \ldots \mathrm{O})=1.8 \AA$ to zero at $r(\mathrm{H} \ldots \mathrm{O})=5.0 \AA$. [Although the hydrogen bond might be considered to be absent for $r(\mathrm{H} \ldots \mathrm{O}) \geqslant 2.5 \AA, 5$ the above variation still allows for some nonbonded interactions at longer distances.] For the external $\mathrm{CO}$ and $\mathrm{NH}$ groups, the presence or absence of hydrogen bonds has a significant effect on the amide II and III frequencies (lowering them when external hydrogen bonds are not included ${ }^{1}$ ). Since these groups would usually be expected to be hydrogen bonded in proteins, we have incorporated the effects of such bonding by placing a hydrogen atom in the appropriate position with respect to the $\mathrm{CO}$ group and an oxygen atom in a comparable position with respect to the $\mathrm{NH}$ group (Fig. 1). These atoms, and $f(\mathrm{H} \ldots \mathrm{O})=0.125 \mathrm{md} \AA^{-1}$, were included in the computations and were kept constant during variations in dihedral angles. Although such values may not exactly match those found in particular proteins, their inclusion should provide a valid measure of the influence of hydrogen bonding on the above amide frequencies. (It should be noted that such hydrogen bonds would not be expected for oligopeptides in nonpolar solvents, and the effects on amide II and III indicated by our earlier calculations ${ }^{1}$ would be expected to prevail.)

The force field for the calculations was taken from our previous work on polypeptide structures. ${ }^{10-12}$ The effects of transition dipole coupling 8,9 were incorporated only for the amide I and II modes. In the amide III mode, coordinates other than $\mathrm{NH}$ in-plane bending make a significant contribution, and as in the case of $\beta$-poly(L-alanine), ${ }^{11}$ the coupling of $\mathrm{NH}$ in-plane bending modes does not have an appreciable effect in perturbing the frequencies. (This is in contrast to the case of polyglycine $1,{ }^{10}$ in which $\mathrm{NH}$ in-plane bending makes comparable contributions to the amide II and III modes.) The positions and orientations of the transition dipole moments with respect to the $\mathrm{CO}$ and $\mathrm{NH}$ bonds were assumed to be the same as in previous work, ${ }^{10,11}$ and all interactions were included. We do not, however, have independent information on the magnitudes of the effective moments, $\Delta \mu_{\text {eff }}$, in the $\beta$-turn as compared with the $\beta$-sheet and $\alpha$-helix structures. We have, therefore, computed the amide $I$ and II frequencies for a range of values of this parameter: $\Delta \mu_{\mathrm{eff}}=0,0.30,0.35$, and $0.45 \mathrm{D}$ for amide I [for $\beta$-poly(L-alanine) we found ${ }^{11}$ that $\Delta \mu_{\mathrm{eff}}=0.37 \mathrm{D}$ ], and $\Delta \mu_{\mathrm{eff}}$ $=0,0.20,0.27$, and $0.40 \mathrm{D}$ for amide II [ the value found for $\beta$-poly (L-alanine) was $\left.\Delta \mu_{\mathrm{eff}}=0.27 \mathrm{D}\right]$.

In the calculations for the structures whose dihedral angles varied from the standard values, we attempted to choose dihedral angles representative of those found in proteins. Our analysis of the structural data on 38 nonhomologous proteins in the Brookhaven National Laboratory Protein Data Bank indicated that the majority of $\beta$-turns of types I and II had average variations in $\phi_{2}$ of up to $-30^{\circ}$, in $\psi_{2}$ and $\phi_{3}$ of up to $\pm 30^{\circ}$, and in $\psi_{3}$ of up 
to $\pm 35^{\circ}$. For type III $\beta$-turns the comparable values were: $\phi_{1}$ up to $-10^{\circ}$, $\psi_{2}$ up to $-25^{\circ}, \phi_{3}$ up to $+15^{\circ}$ and $-25^{\circ}$, and $\psi_{3}$ up to $+15^{\circ}$ and $-20^{\circ}$. We therefore computed the normal vibration frequencies of such $\beta$-turns with each angle varied from the standard value by the above amounts. Since, as indicated above, transition dipole coupling also involves peptide groups 1 and 5 , it was desirable to investigate the effects of variations in $(\varphi, \psi)_{1}$ and $(\phi, \psi)_{4}$ on the characteristic $\beta$-turn frequencies. Examination of the protein data led us to select for normal mode calculations the following additional values of these dihedral angles: $\phi_{1}=-60^{\circ}, 120^{\circ} ; \psi_{1}=-60^{\circ},-120^{\circ} ; \phi_{4}=$ $-60^{\circ}, 120^{\circ}$; and $\psi_{4}=-60^{\circ},-120^{\circ}$ for types I and II $\beta$-turns; and $\phi_{1}=-60^{\circ}$; $\psi_{1}=40^{\circ},-40^{\circ} ; \phi_{4}=-80^{\circ},-45^{\circ}$; and $\psi_{4}=60^{\circ},-40^{\circ}$ for type III $\beta$-turns. In these computations external hydrogen bonds remained constant and internal hydrogen bonds varied according to the algorithm discussed above.

In the above calculations the force field was assumed to remain constant when the structure was varied. Frequency shifts therefore arise from changes in mechanical coupling ( $\mathrm{G}$ matrix elements) and from variations in transition dipole coupling. This may not, of course, actually be the case. Force constants may also change with structure, and there is some evidence for such changes in going from $\beta$-poly(L-alanine) to $\alpha$-poly(L-alanine). ${ }^{12}$ While some of these changes may be associated with differences in hydrogen-bond strength between these structures, others may be inherently related to the dependence of force constants on internal structure. There is no detailed information available at the present time that would permit this factor to be taken into account in the calculation, and we have therefore proceeded on the (nevertheless reasonable) assumption that the main influence on the frequencies arises from structural differences. There is much evidence for the validity of this concept, an example of which is the rotational isomers of chlorinated hydrocarbons. ${ }^{13}$

\section{RESULTS}

We present in Tables I-III the calculated frequencies for standard $\beta$-turns of types I-III, respectively, in the absence of transition dipole coupling. The frequencies for $\mathrm{NH}$ stretch, $\mathrm{CH}_{3}$ asymmetric stretch, $\mathrm{CH}_{3}$ symmetric stretch, $\mathrm{C}^{\wedge} \mathrm{H}^{*}$ stretch, $\mathrm{CH}_{3}$ asymmetric bend, and $\mathrm{CH}_{3} \mathrm{sym}$ metric bend are not given explicitly, since they are not particularly distinctive; only the frequency intervals and numbers of normal modes are given. For the other frequencies, all contributions to the potential energy equal to or greater than $10 \%$ are included. The internal coordinates correspond to the structure in Fig. 1 and are the same as those used in our previous studies. ${ }^{10-12}$ The $\mathrm{C}$ and $\mathrm{N}$ atoms of the peptide groups are numbered the same as the labeled $\mathrm{O}$ and $\mathrm{H}$ atoms of these groups in Fig. 1. The $\mathrm{H}^{\mathrm{r}}$ atoms, and the $\mathrm{CH}_{3}$ groups attached to the $\mathrm{C}^{\alpha}$ atoms, have the same number as the corresponding $\mathrm{C}^{\alpha}$ atoms.

As developed in our earlier work, ${ }^{8-12}$ the observed splittings in the amide 
TABLE I

Calculated Frequencies of a Type $I \beta$-Turn of $\mathrm{CH}_{3}-\mathrm{CO}-(\mathrm{Ala})_{4}-\mathrm{NH}-\mathrm{CH}_{3}$

\begin{tabular}{|c|c|}
\hline $\begin{array}{l}\text { Frequency } \\
\quad\left(\mathrm{cm}^{1}\right)\end{array}$ & Potential Energy Distributiona \\
\hline $3275-3265$ & NH str: 5 modes \\
\hline $2979-2976$ & $\mathrm{CH}_{3}$ asym str: 12 modes \\
\hline $2942-2941$ & $\mathrm{CH}_{3}$ sym str: 6 modes \\
\hline $2876-2873$ & $\mathrm{C}^{\alpha} \mathrm{H}^{\alpha}$ str: 4 modes \\
\hline 1676 & $\mathrm{C}=\mathrm{O}(5) \operatorname{str}(68), \mathrm{CN}(5) \operatorname{str}(13)$ \\
\hline 1673 & $\mathrm{C}=\mathrm{O}(2) \operatorname{str}(51), \mathrm{C}=\mathrm{O}(1) \operatorname{str}(22)$ \\
\hline 1671 & $\mathrm{C}=\mathrm{O}(1) \operatorname{str}(49), \mathrm{C}=\mathrm{O}(2) \operatorname{str}(23)$ \\
\hline 1666 & $\mathrm{C}=\mathrm{O}(3) \operatorname{str}(56), \mathrm{C}=\mathrm{O}(4) \operatorname{str}(20)$ \\
\hline 1665 & $\mathrm{C}=\mathrm{O}(4) \operatorname{str}(55), \mathrm{C}=\mathrm{O}(3) \operatorname{str}(21)$ \\
\hline 1579 & $\mathrm{NH}(5)$ ipb(53), $\mathrm{C}^{\prime \prime} \mathrm{H}_{3}$ sym bend(22) \\
\hline 1569 & $\mathrm{NH}(4)$ ipb(49), CN(4) str(23) \\
\hline 1567 & $\mathrm{NH}(1) \operatorname{ipb}(43), \mathrm{CN}(1) \operatorname{str}(20)$ \\
\hline 1554 & $\mathrm{NH}(2) \mathrm{ipb}(51), \mathrm{CN}(2) \operatorname{str}(23)$ \\
\hline 1540 & $\mathrm{NH}(3)$ ipb(47), CN(3) str(29) \\
\hline $1468-1451$ & $\mathrm{CH}_{3}$ asym bend: 12 modes \\
\hline $1380-1374$ & $\mathrm{CH}_{3}$ sym bend: 6 modes \\
\hline 1371 & $\mathrm{H}^{\alpha}(1)$ bend $1(41), \mathrm{C}^{\prime} \mathrm{H}_{3}$ sym bend(14) \\
\hline 1371 & $H^{\alpha}(3)$ bend $1(22), H^{*}(4)$ bend $1(19)$ \\
\hline 1356 & $H^{\alpha}(3)$ bend $1(28), H^{\alpha}(4)$ bend $1(27)$ \\
\hline 1353 & $\mathrm{H}^{\propto}(2)$ bend $1(58), \mathrm{CH}_{3}(1)$ sym bend(11) \\
\hline 1331 & $\mathrm{NH}(1)$ ipb(28), $\mathrm{C}^{\prime} \mathrm{C} \operatorname{str}(18), \mathrm{CN}(1) \operatorname{str}(16), \mathrm{C}=\mathrm{O}(1)$ ipb(13) \\
\hline 1324 & $\begin{array}{l}\mathrm{NH}(4) \text { ipb(23), } \mathrm{C}^{\alpha}(3) \mathrm{C} \operatorname{str}(15), \mathrm{CN}(4) \operatorname{str}(15), \mathrm{H}^{\alpha}(3) \text { bend } \\
1(11)\end{array}$ \\
\hline 1305 & $\mathrm{NH}(3)$ ipb(29), $\mathrm{C}^{\alpha}(2) \mathrm{C} \operatorname{str}(15), \mathrm{CN}(3) \operatorname{str}(10)$ \\
\hline 1299 & $\begin{array}{l}\mathrm{NH}(2) \text { ipb }(28), \mathrm{C}^{\alpha}(1) \mathrm{C} \operatorname{str}(11), \mathrm{NH}(3) \text { ipb(11), CN(2) str(10), } \\
\mathrm{H}^{\prime x}(1) \text { bend } 1(10)\end{array}$ \\
\hline 1293 & $\begin{array}{l}\text { NH(5) ipb(37), } \mathrm{C}^{\alpha}(4) \mathrm{C} \operatorname{str}(17), \mathrm{H}^{\alpha}(4) \text { bend } 1(13), \mathrm{CN}(5) \\
\quad \operatorname{str}(12), \mathrm{C}=\mathrm{O}(5) \text { ipb }(10)\end{array}$ \\
\hline 1212 & $\mathrm{NC}^{\alpha}(2) \operatorname{str}(30), \mathrm{NC}^{\alpha}(1) \operatorname{str}(22)$ \\
\hline 1199 & $\mathrm{NC}^{\alpha}(3) \operatorname{str}(31), \mathrm{NC}^{\alpha}(4) \operatorname{str}(11)$ \\
\hline 1189 & $\begin{array}{l}\mathrm{NC}^{\alpha}(4) \operatorname{str}(30), \mathrm{NC}^{\alpha}(3) \operatorname{str}(14), \mathrm{C}^{\alpha}(3) \mathrm{C} \operatorname{str}(10), \mathrm{CH}_{3}(3) \text { rock } \\
2(10)\end{array}$ \\
\hline 1166 & $\mathrm{NC}^{\alpha(}(1) \operatorname{str}(25), \mathrm{NC}^{\alpha}(2) \operatorname{str}(14)$ \\
\hline 1116 & $N C^{\prime \prime} \operatorname{str}(19), C^{\alpha}(4) C^{\beta} \operatorname{str}(14)$ \\
\hline 1113 & $\mathrm{CH}_{3}(3)$ rock $1(18), \mathrm{C}^{\prime \prime} \mathrm{H}_{3}$ rock $2(16), \mathrm{C}^{\alpha}(4) \mathrm{C}^{\beta} \operatorname{str}(10)$ \\
\hline 1111 & $\mathrm{C}^{\prime} \mathrm{H}_{3}$ rock $1(32), \mathrm{C}^{\prime} \mathrm{H}_{3}$ rock $2(11), \mathrm{C}^{\alpha}(1) \mathrm{C}^{\beta} \operatorname{str}(10)$ \\
\hline 1106 & $\begin{array}{l}\mathrm{C}^{\prime \prime} \mathrm{H}_{3} \text { rock } 2(29), \mathrm{CH}_{3}(3) \text { rock } 1(18), \mathrm{C}^{\prime \prime} \mathrm{H}_{3} \text { rock } 1(11), \mathrm{C}^{\alpha}(4) \mathrm{C}^{\beta} \\
\operatorname{str}(10)\end{array}$ \\
\hline 1102 & $\mathrm{CH}_{3}(2)$ rock $1(20), \mathrm{C}^{\alpha}(3) \mathrm{C}^{\beta} \operatorname{str}(13)$ \\
\hline 1095 & $\mathrm{CH}_{3}(1)$ rock $1(20), \mathrm{CH}_{3}(2)$ rock $1(17), \mathrm{C}^{\alpha}(2) \mathrm{C}^{\beta} \operatorname{str}(17)$ \\
\hline 1091 & $\mathrm{C}^{\alpha}(1) \mathrm{C}^{\beta} \operatorname{str}(18), \mathrm{C}^{\alpha}(2) \mathrm{C}^{\beta} \operatorname{str}(16), \mathrm{CH}_{3}(1)$ rock $1(10)$ \\
\hline 1082 & $\mathrm{C}^{\prime \alpha}(2) \mathrm{C}^{\beta} \operatorname{str}(13), \mathrm{CH}_{3}(2)$ rock $2(13), \mathrm{C}^{\alpha}(3) \mathrm{C}^{\alpha \beta} \operatorname{str}(12)$ \\
\hline 1078 & $\mathrm{C}^{\prime \prime} \mathrm{H}_{3}$ rock $1(52), \mathrm{C}^{\prime \prime} \mathrm{H}_{3}$ rock $2(21), \mathrm{C}^{\prime \prime} \mathrm{H}_{3}$ asym bend $1(10)$ \\
\hline 1070 & $\mathrm{C}^{\alpha}(3) \mathrm{C}^{\beta} \operatorname{str}(16), \mathrm{C}^{\alpha}(2) \mathrm{C}^{\beta} \operatorname{str}(13)$ \\
\hline 1049 & $\mathrm{CH}_{3}(4)$ rock $1(56), \mathrm{CH}_{3}(4)$ rock $2(23)$ \\
\hline 1022 & $\mathrm{NC}^{\prime \prime} \operatorname{str}(40), \mathrm{CH}_{3}(3)$ rock $2(29)$ \\
\hline 1016 & $\mathrm{CH}_{3}(4)$ rock $2(43), \mathrm{CH}_{3}(4)$ rock $1(20)$ \\
\hline 994 & $\mathrm{H}^{*}(1)$ bend $2(44), \mathrm{C}^{\alpha}(1) \mathrm{C}^{\beta} \operatorname{str}(10)$ \\
\hline
\end{tabular}




\begin{tabular}{|c|c|}
\hline $\begin{array}{l}\text { Frequency } \\
\left(\mathrm{cm}^{2}\right)\end{array}$ & Potential Energy Distribution ${ }^{a}$ \\
\hline 990 & $H^{\alpha}(4)$ bend $2(59), C^{\alpha}(4) C^{\beta} \operatorname{str}(12)$ \\
\hline 984 & $\mathrm{H}^{\star}(3)$ bend $2(31), \mathrm{H}^{\star}(2)$ bend $2(18), \mathrm{CH}_{3}(2)$ rock $1(11)$ \\
\hline 983 & $H^{\alpha}(3)$ bend $2(28), H^{\alpha}(2)$ bend $2(21), H^{\alpha}(1)$ bend $2(10)$ \\
\hline 977 & $\mathrm{H}^{\alpha}(2)$ bend $2(20), \mathrm{CH}_{3}(1)$ rock $2(20), \mathrm{NC}^{\alpha}(2) \operatorname{str}(10)$ \\
\hline 967 & $\mathrm{CH}_{3}(2)$ rock $2(21), \mathrm{NC}^{\alpha}(3) \operatorname{str}(13), \mathrm{H}^{\alpha}(3)$ bend $2(10)$ \\
\hline 954 & $\mathrm{CH}_{3}(1)$ rock $2(13), \mathrm{NC}^{\alpha}(1) \operatorname{str}(10)$ \\
\hline 938 & $\begin{array}{l}\mathrm{NC}^{\alpha}(4) \operatorname{str}(22), \mathrm{NC}^{\prime \prime} \operatorname{str}(11), \mathrm{C}^{\alpha}(4) \mathrm{C}^{\beta} \operatorname{str}(11), \mathrm{CH}_{3}(3) \text { rock } \\
2(10)\end{array}$ \\
\hline 913 & $\mathrm{CN}(1) \operatorname{str}(23), \mathrm{C}^{\prime} \mathrm{H}_{3}$ rock $1(11), \mathrm{CNC}^{\alpha}(1) \operatorname{def}(11)$ \\
\hline 898 & $\mathrm{CN}(4) \operatorname{str}(12), \mathrm{CNC}^{\alpha}(4) \operatorname{def}(11)$ \\
\hline 897 & $\mathrm{C}^{x}(1) \mathrm{C} \operatorname{str}(13), \mathrm{CN}(2) \operatorname{str}(13), \mathrm{C}=\mathrm{O}(2) \operatorname{str}(10)$ \\
\hline 879 & $\mathrm{CN}(3) \operatorname{str}(16)$ \\
\hline 855 & $\mathrm{CN}(5) \operatorname{str}(15), \mathrm{C}^{\alpha}(4) \mathrm{C} \operatorname{str}(14)$ \\
\hline 762 & $\mathrm{C}=\mathrm{O}(2)$ opb(17), $\mathrm{C}=\mathrm{O}(3) \circ \mathrm{pb}(16)$ \\
\hline 754 & $\mathrm{C}=\mathrm{O}(4)$ opb(13), $\mathrm{C}=\mathrm{O}(5)$ opb(10) \\
\hline 737 & $\mathrm{C}=\mathrm{O}(5)$ opb(22), $\mathrm{C}=\mathrm{O}(3)$ opb(14) \\
\hline 721 & $\mathrm{C}=\mathrm{O}(4)$ opb $(20), \mathrm{C}=\mathrm{O}(3)$ opb(13) \\
\hline 697 & $\mathrm{C}=\mathrm{O}(2) \mathrm{ipb}(16), \mathrm{C}=\mathrm{O}(2) \mathrm{opb}(13), \mathrm{C}^{\alpha}(1) \mathrm{C} \operatorname{str}(10)$ \\
\hline 683 & $\mathrm{C}=\mathrm{O}(5)$ ipb(13), $\mathrm{C}=\mathrm{O}(4)$ opb(11), $\mathrm{C}=\mathrm{O}(5)$ opb(11) \\
\hline 648 & $\mathrm{C}=\mathrm{O}(1)$ opb(13), $\mathrm{C}^{\alpha}(1) \mathrm{CN} \operatorname{def}(12)$ \\
\hline 645 & $\mathrm{C}=\mathrm{O}(5) \mathrm{ipb}(11)$ \\
\hline 633 & $\mathrm{CN}(4)$ tor $(23)$ \\
\hline 613 & $\mathrm{C}=\mathrm{O}(1)$ opb $(40), \mathrm{CN}(1)$ tor $(33)$ \\
\hline 597 & $\begin{array}{l}\mathrm{C}=\mathrm{O}(1) \operatorname{ipb}(24), \mathrm{CN}(4) \text { tor }(13), \mathrm{C}^{\prime} \mathrm{C} \operatorname{str}(10), \mathrm{CN}(1) \text { tor }(10), \\
\mathrm{CN}(3) \operatorname{tor}(10)\end{array}$ \\
\hline 595 & $\begin{array}{l}\mathrm{CN}(4) \text { tor }(30), \mathrm{CN}(1) \text { tor }(17), \mathrm{CN}(2) \text { tor }(17), \mathrm{CN}(3) \text { tor }(12) \text {, } \\
\mathrm{NH}(4) \text { opb }(12)\end{array}$ \\
\hline 584 & $\mathrm{CN}(1)$ tor $(49), \mathrm{H}^{\alpha}(1)$ bend $2(16), \mathrm{C}=\mathrm{O}(1)$ opb(10) \\
\hline 575 & $\mathrm{CN}(3)$ tor $(50), \mathrm{NH}(3)$ opb $(23), \mathrm{CN}(5)$ tor $(19), \mathrm{NH}(5)$ opb(11) \\
\hline 574 & $\mathrm{CN}(2)$ tor $(44), \mathrm{NH}(2)$ opb $(26), \mathrm{NH}(5)$ opb(14), $\mathrm{CN}(5)$ tor(13) \\
\hline 570 & $\mathrm{NH}(5)$ opb (25), CN(2) tor(19), NH(2) opb(12), NH(3) opb(11) \\
\hline 531 & $\mathrm{C}=\mathrm{O}(3) \mathrm{ipb}(11), \mathrm{C}^{\prime} \mathrm{CN} \operatorname{def}(10)$ \\
\hline 500 & $\mathrm{NC}^{\alpha}(2) \mathrm{C} \operatorname{def}(15), \mathrm{C}^{\prime} \mathrm{CN} \operatorname{def}(10)$ \\
\hline 473 & $\mathrm{H} \ldots \mathrm{O}(4) \operatorname{str}(76)$ \\
\hline 471 & $\mathrm{H} \ldots \mathrm{O}(3) \mathrm{str}(80)$ \\
\hline 469 & $\mathrm{H} \ldots \mathrm{O}(1) \operatorname{str}(62), \mathrm{H}(4) \ldots \mathrm{O}(2) \operatorname{str}(11)$ \\
\hline 443 & $\mathrm{C}^{\beta}(3)$ bend $1(22)$ \\
\hline 412 & $\mathrm{C}^{\prime} \mathrm{CN} \operatorname{def}(22), \mathrm{C}^{\beta}(2)$ bend $1(17)$ \\
\hline 403 & $\mathrm{NC}^{\alpha}(3) \mathrm{C} \operatorname{def}(34), \mathrm{NC}^{\alpha}(2) \mathrm{C} \operatorname{def}(10)$ \\
\hline 370 & $\mathrm{C}^{\beta}(3)$ bend $2(14), \mathrm{C}^{\beta}(4)$ bend $1(11)$ \\
\hline 350 & $\mathrm{C}^{\beta}(2)$ bend $1(21), \mathrm{C}=\mathrm{O}(2)$ ipb(11) \\
\hline 332 & $\mathrm{C}^{\beta}(2)$ bend $2(20), \mathrm{C}=\mathrm{O}(3)$ ipb(14) \\
\hline 313 & $C^{\beta}(4)$ bend $2(25)$ \\
\hline 308 & $\mathrm{C}^{\beta}(1)$ bend $2(18), \mathrm{NC}^{\alpha}(1) \mathrm{C} \operatorname{def}(11)$ \\
\hline 298 & $\mathrm{C}^{\beta}(3)$ bend $2(24)$ \\
\hline 277 & $C N C^{\prime \prime} \operatorname{def}(18), C=O(5) \operatorname{ipb}(10)$ \\
\hline 263 & $\mathrm{C}^{\alpha}(2) \mathrm{C}^{\beta}$ tor $(13)$ \\
\hline 257 & $\begin{array}{c}\mathrm{C}^{\alpha}(4) \mathrm{C}^{\beta} \operatorname{tor}(34), \mathrm{C}^{\alpha}(3)^{\beta} \operatorname{tor}(11), \mathrm{CNC}^{\prime \prime} \operatorname{def}(10), \mathrm{C}^{\beta}(2) \text { bend } \\
2(10)\end{array}$ \\
\hline 250 & $\mathrm{C}^{\alpha}(2) \mathrm{C}^{\beta} \operatorname{tor}(44), \mathrm{C}^{\alpha}(1) \mathrm{C}^{\beta} \operatorname{tor}(21), \mathrm{C}^{\alpha \dot{x}}(3) \mathrm{C}^{\beta} \operatorname{tor}(17)$ \\
\hline 249 & $\mathrm{C}^{\alpha}(3) \mathrm{C}^{\beta}$ tor $(56), \mathrm{C}^{\alpha}(1) \mathrm{C}^{\beta}$ tor $(29)$ \\
\hline
\end{tabular}




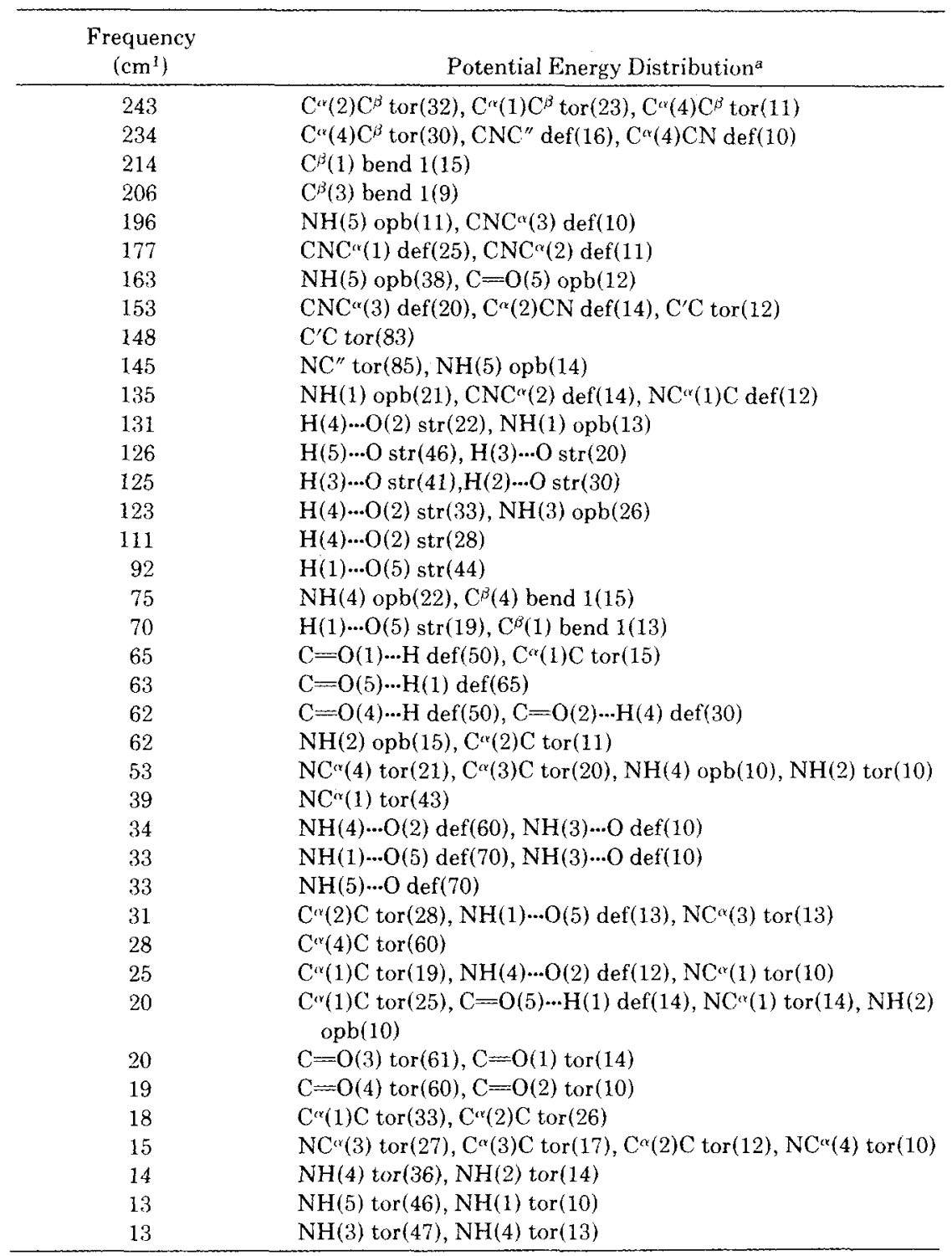

\footnotetext{
a Only contributions equal to or greater than $10 \%$ are included. Abbreviations used: asym, asymmetric; def, deformation; ipb, in-plane bend; opb, out-of-plane bend; str, stretch; sym, symmetric; tor, torsion.
}

I and II modes cannot be accounted for satisfactorily without incorporating the effects of transition dipole coupling. The results of doing so for the type I-III turns are shown in Table IV; the amide III modes are included for convenience in making comparisons between these three characteristic amide modes of the $\beta$-turns. Results are shown for several values of the effective dipole moment, $\Delta \mu_{\text {eff }}$, since the values derived for the $\beta$-sheet 
TABLE II

Calculated Frequencies of a Type II $\beta$-Turn of $\mathrm{CH}_{3}-\mathrm{CO}-(\mathrm{Ala})_{2}-\mathrm{Gly}-\mathrm{Ala}-\mathrm{NH}-\mathrm{CH}_{3}$

\begin{tabular}{|c|c|}
\hline $\begin{array}{c}\text { Frequency } \\
\left(\mathrm{cm}^{-1}\right)\end{array}$ & Potential Energy Distribution ${ }^{a}$ \\
\hline $3276-3264$ & NH str: 5 modes \\
\hline $2979-2978$ & $\mathrm{CH}_{3}$ asym str: 10 modes \\
\hline 2941 & $\mathrm{CH}_{3}$ sym str: 5 modes \\
\hline 2932 & $\mathrm{CH}_{2}$ asym $\operatorname{str}(97)$ \\
\hline $2876-2874$ & $\mathrm{C}^{*} \mathrm{H}^{*}$ str: 3 modes \\
\hline 2863 & $\mathrm{CH}_{2}$ sym str $(96)$ \\
\hline 1676 & $\mathrm{C}=\mathrm{O}(5) \operatorname{str}(64), \mathrm{CN}(5) \operatorname{str}(12)$ \\
\hline 1675 & $\mathrm{C}=\mathrm{O}(3) \mathrm{str}(65), \mathrm{CN}(3) \mathrm{str}(12)$ \\
\hline 1674 & $\mathrm{C}=\mathrm{O}(2) \operatorname{str}(55), \mathrm{C}=\mathrm{O}(1) \operatorname{str}(14)$ \\
\hline 1671 & $\mathrm{C}=\mathrm{O}(1) \operatorname{str}(55), \mathrm{C}=\mathrm{O}(2) \operatorname{str}(16)$ \\
\hline 1665 & $\mathrm{C}=\mathrm{O}(4) \operatorname{str}(75), \mathrm{C}^{\star} \mathrm{CN}(4) \operatorname{def}(12)$ \\
\hline 1578 & $\mathrm{NH}(5)$ ipb(32), $\mathrm{C}^{\prime \prime} \mathrm{H}_{33}$ sym bend(26) \\
\hline 1568 & NH(4) ipb(21), NH(1) ipb(20) \\
\hline 1567 & $\mathrm{NH}(1) \operatorname{ipb}(33), \mathrm{NH}(4)$ ipb(23) \\
\hline 1563 & NH(3) ipb(20), CN(3) str(12) \\
\hline 1553 & $\mathrm{NH}(2)$ ipb(23), CN(2) str(21) \\
\hline $1468-1462$ & $\mathrm{CH}_{3}$ asym bend: 10 modes \\
\hline 1457 & $\mathrm{CH}_{2}$ bend $(86)$ \\
\hline 1391 & $\mathrm{CH}_{2}$ wag $(76)$ \\
\hline 1391 & $H^{\alpha}(3)$ bend $1(21), H^{\alpha}(2)$ bend $1(13)$ \\
\hline $1390-1378$ & $\mathrm{CH}_{3}$ sym bend: 5 modes \\
\hline 1377 & $H^{\alpha}(1)$ bend $1(26), H^{\alpha}(3)$ bend $1(10)$ \\
\hline 1330 & $\mathrm{NH}(1)$ ipb(28), $\mathrm{C}^{\prime} \mathrm{C} \operatorname{str}(17)$ \\
\hline 1329 & $\mathrm{NH}(4) \operatorname{ipb}(22), \mathrm{C}(3) \mathrm{C} \operatorname{str}(18)$ \\
\hline 1303 & NH(2) ipb(19), NH(3) ipb(12) \\
\hline 1297 & $\mathrm{NH}(3)$ ipb(21), $\mathrm{NH}(2)$ ipb(13) \\
\hline 1292 & $\mathrm{NH}(5)$ ipb(37), $\mathrm{C}^{\alpha}(4) \mathrm{C} \operatorname{str}(16)$ \\
\hline 1250 & $\mathrm{CH}_{2}$ twist $(73)$ \\
\hline 1218 & $\mathrm{NC}^{\alpha}(2) \operatorname{str}(24), \mathrm{NC}^{\wedge}(1) \operatorname{str}(15)$ \\
\hline 1195 & $\mathrm{NC}^{\alpha}(4) \operatorname{str}(38), \mathrm{CH}_{3}(2) \operatorname{rock} 1(10)$ \\
\hline 1184 & $\mathrm{NC}^{\circ}(1) \operatorname{str}(19), \mathrm{NC}^{\circ}(3) \operatorname{str}(17)$ \\
\hline 1153 & $N^{\alpha}(2) \operatorname{str}(19), N^{\alpha}(1) \operatorname{str}(11)$ \\
\hline 1127 & $\mathrm{CH}_{3}(2)$ rock $1(13), \mathrm{H}^{\alpha}(3)$ bend $2(10)$ \\
\hline 1110 & $\mathrm{C}^{\prime} \mathrm{H}_{3}$ rock $1(33), \mathrm{CH}_{3}(1)$ rock $2(11)$ \\
\hline 1107 & $\mathrm{NC}^{\prime \prime} \operatorname{str}(12), \mathrm{C}^{\prime \prime} \mathrm{H}_{3}$ rock $2(10)$ \\
\hline 1106 & $\mathrm{CH}_{3}(1) \operatorname{rock} \lambda(28), \mathrm{C}^{\alpha}(2) \mathrm{C}^{\beta} \operatorname{str}(11)$ \\
\hline 1102 & $\mathrm{CH}_{3}(2) \operatorname{rock} 1(13), \mathrm{CH}_{3}(1) \operatorname{rock} 2(13)$ \\
\hline 1096 & $\mathrm{C}^{\alpha}(1) \mathrm{C}^{\beta} \operatorname{str}(25), \mathrm{C}^{\prime} \mathrm{H}_{3} \operatorname{rock} 2(13)$ \\
\hline 1084 & $\mathrm{C}^{\prime \prime} \mathrm{H}_{3}$ rock $1(56), \mathrm{C}^{\prime \prime} \mathrm{H}_{3}$ rock $2(22)$ \\
\hline 1079 & $\mathrm{CH}_{3}(4)$ rock $1(56), \mathrm{CH}_{3}(4)$ rock $2(22)$ \\
\hline 1063 & 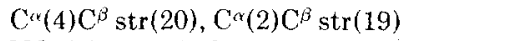 \\
\hline 1049 & $\mathrm{NC}^{\circ}(3) \operatorname{str}(37), \mathrm{CH}_{3}(2)$ rock $1(16)$ \\
\hline 1022 & $\mathrm{NC}^{\prime \prime} \operatorname{str}(40), \mathrm{CH}_{3}(1)$ rock $1(11)$ \\
\hline 1016 & $\mathrm{CH}_{3}(4)$ rock $2(43), \mathrm{CH}_{3}(4)$ rock $1(19)$ \\
\hline 1007 & $\mathrm{CH}_{2} \operatorname{rock}(50), \mathrm{C}=\mathrm{O}(4) \operatorname{str}(12)$ \\
\hline 994 & $\mathrm{H}^{\alpha}(1)$ bend $2(51), \mathrm{C}^{\alpha}(1) \mathrm{C}^{\beta} \operatorname{str}(11)$ \\
\hline 990 & $\mathrm{H}^{\alpha}(4)$ bend $2(58), \mathrm{C}^{\alpha}(4) \mathrm{C}^{\beta} \operatorname{str}(11)$ \\
\hline 981 & $\mathrm{CH}_{3}(2)$ rock $2(11), \mathrm{CH}_{3}(1)$ rock $2(10)$ \\
\hline
\end{tabular}




\begin{tabular}{|c|c|}
\hline $\begin{array}{l}\text { Frequency } \\
\left(\mathrm{cm}^{-1}\right)\end{array}$ & Potential Energy Distribution $^{a}$ \\
\hline 976 & $\mathrm{H}^{\prime \prime}(2)$ bend $2(50), \mathrm{C}^{\alpha}(2) \mathrm{C}^{\beta} \operatorname{str}(14)$ \\
\hline 944 & $\mathrm{NC}^{\alpha}(2) \operatorname{str}(12), \mathrm{CH}_{3}(1)$ rock $2(10)$ \\
\hline 935 & $\mathrm{NC}^{\alpha}(4) \operatorname{str}(15)$ \\
\hline 913 & $\mathrm{CN}(1) \operatorname{str}(23), \mathrm{CNC}^{\alpha}(1) \operatorname{def}(11)$ \\
\hline 895 & $\mathrm{C}^{\alpha}(1) \mathrm{C} \operatorname{str}(13), \mathrm{CN}(2) \operatorname{str}(10)$ \\
\hline 890 & $\mathrm{CNC}^{\alpha}(4) \operatorname{def}(8)$ \\
\hline 883 & $\mathrm{CN}(3) \operatorname{str}(14), \mathrm{C}^{\mathrm{c}}(2) \mathrm{C} \operatorname{str}(12)$ \\
\hline 850 & $\mathrm{CN}(5) \operatorname{str}(13), \mathrm{C}^{\alpha}(4) \mathrm{C} \operatorname{str}(11)$ \\
\hline 768 & $\mathrm{C}=\mathrm{O}(2)$ opb(14), CN(2) tor $(11)$ \\
\hline 747 & $\mathrm{C}=\mathrm{O}(5)$ opb $(16), \mathrm{C}=\mathrm{O}(4)$ opb(12) \\
\hline 734 & $\mathrm{C}=\mathrm{O}(5)$ opb(16), $\mathrm{C}=\mathrm{O}(3) \mathrm{opb}(11)$ \\
\hline 724 & $\mathrm{C}=\mathrm{O}(3)$ opb $(31), \mathrm{C}=\mathrm{O}(4)$ opb(12) \\
\hline 715 & $\mathrm{C}=\mathrm{O}(4)$ opb(12), $\mathrm{C}=\mathrm{O}(3) \mathrm{ipb}(11)$ \\
\hline 684 & $\mathrm{C}=\mathrm{O}(2)$ opb(17), $\mathrm{C}=\mathrm{O}(2)$ ipb(10) \\
\hline 674 & $\mathrm{C}=\mathrm{O}(5) \mathrm{ipb}(17), \mathrm{C}=\mathrm{O}(5) \mathrm{opb}(15)$ \\
\hline 644 & $\mathrm{NH}(4)$ opb $(20), \mathrm{CN}(4)$ tor $(20), \mathrm{CH}_{2} \operatorname{rock}(13)$ \\
\hline 643 & $\mathrm{C}=\mathrm{O}(1)$ opb(17), $\mathrm{C}^{\alpha}(1) \mathrm{CN} \operatorname{def}(11), \mathrm{CH}_{2} \operatorname{wag}(10)$ \\
\hline 619 & $\mathrm{CN}(1)$ tor $(57), \mathrm{C}=\mathrm{O}(1)$ opb $(24)$ \\
\hline 607 & $\mathrm{CN}(1)$ tor $(38), \mathrm{NH}(1)$ opb(29) \\
\hline 600 & $\mathrm{C}=\mathrm{O}(1) \mathrm{ipb}(21), \mathrm{CN}(3)$ tor(14), $\mathrm{CH}_{2} \operatorname{rock}(13)$ \\
\hline 594 & $\mathrm{CN}(4)$ tor $(46), \mathrm{NH}(4)$ opb(19) \\
\hline 588 & $\mathrm{CN}(3)$ tor $(44), \mathrm{NH}(3)$ opb $(40)$ \\
\hline 572 & $\mathrm{CN}(5)$ tor(85), NH(5) opb(13) \\
\hline 571 & $\mathrm{CN}(2)$ tor $(59), \mathrm{NH}(2)$ opb(39) \\
\hline 544 & $\mathrm{C}^{\prime} \mathrm{CN} \operatorname{def}(17), \mathrm{C}=\mathrm{O}(1) \mathrm{ipb}(11)$ \\
\hline 481 & $\mathrm{C}^{\alpha}(4) \mathrm{CN} \operatorname{def}(10)$ \\
\hline 477 & $\mathrm{H} \ldots \mathrm{O}(4) \operatorname{str}(62), \mathrm{H}(4) \ldots \mathrm{O}(2) \operatorname{str}(12)$ \\
\hline 473 & $\mathrm{H} \cdots \mathrm{O}(1) \operatorname{str}(51), \mathrm{H}(1) \cdots \mathrm{O}(5) \operatorname{str}(10)$ \\
\hline 472 & $\mathrm{H} \ldots \mathrm{O}(3) \operatorname{str}(81)$ \\
\hline 434 & $\mathrm{C}^{\beta}(2)$ bend $2(13), \mathrm{C}^{\prime} \mathrm{CN} \operatorname{def}(11)$ \\
\hline 424 & $\mathrm{NC}^{\alpha}(3) \mathrm{C} \operatorname{def}(16)$ \\
\hline 410 & $\mathrm{C}^{\beta}(1)$ bend $1(17), \mathrm{C}^{\prime} \mathrm{CN} \operatorname{def}(13)$ \\
\hline 342 & $\mathrm{C}=\mathrm{O}(2) \operatorname{ipb}(10), \mathrm{CNC}^{\alpha}(2) \operatorname{def}(10)$ \\
\hline 318 & $\mathrm{C}^{\beta}(1)$ bend $2(27), \mathrm{NC}^{\alpha}(2) \mathrm{C} \operatorname{def}(19)$ \\
\hline 312 & $\mathrm{C}^{\beta}(4)$ bend $2(27), \mathrm{NC}^{\alpha}(4) \operatorname{def}(14)$ \\
\hline 287 & $\mathrm{NC}^{\alpha}(1) \mathrm{C} \operatorname{def}(11), \mathrm{NC}^{\alpha}(2) \mathrm{C} \operatorname{def}(10)$ \\
\hline 280 & $\mathrm{C}^{\beta}(2)$ bend $2(22), \mathrm{C}^{\beta}(2)$ bend $1(10)$ \\
\hline 261 & $\mathrm{C}^{\alpha}(4) \mathrm{C}^{\beta} \operatorname{tor}(24), \mathrm{CNC}^{\prime \prime} \operatorname{def}(19)$ \\
\hline 256 & $\mathrm{C}^{\alpha}(1) \mathrm{C}^{\beta}$ tor $(26), \mathrm{C}^{\beta}(2)$ bend $1(15)$ \\
\hline 247 & $\mathrm{C}^{\alpha}(2) \mathrm{C}^{\beta}$ tor $(58), \mathrm{C}^{\alpha}(4) \mathrm{C}^{\beta} \operatorname{tor}(12)$ \\
\hline 241 & $\mathrm{C}^{\alpha}(1) \mathrm{C}^{\beta} \operatorname{tor}(40)$ \\
\hline 237 & $\mathrm{C}^{\alpha}(4) \mathrm{C}^{\beta}$ tor $(37), \mathrm{C}^{\alpha}(2) \mathrm{C}^{\beta} \operatorname{tor}(12)$ \\
\hline 220 & $C^{\beta}(1)$ bend $1(13)$ \\
\hline 209 & NH(5) opb $(20), C^{\beta}(4)$ bend $1(18)$ \\
\hline 204 & $\mathrm{C}^{\beta}(2)$ bend $1(10)$ \\
\hline 171 & $\mathrm{CNC}^{\alpha}(1) \operatorname{def}(30), \mathrm{NH}(2)$ opb $(10)$ \\
\hline 165 & $\mathrm{NH}(5)$ opb(47), $\mathrm{C}=\mathrm{O}(5)$ opb(13) \\
\hline 149 & $\mathrm{C}^{\prime} \mathrm{C}$ tor $(93)$ \\
\hline 145 & $\mathrm{NC}^{\prime \prime} \operatorname{tor}(86), \mathrm{NH}(5)$ opb(11) \\
\hline 140 & $\mathrm{CNC}^{\alpha \times}(3) \operatorname{def}(27)$ \\
\hline 135 & $\mathrm{NH}(1)$ opb $(22), \mathrm{CNC}^{\alpha}(2) \operatorname{def}(15)$ \\
\hline
\end{tabular}




\begin{tabular}{|c|c|}
\hline $\begin{array}{l}\text { Frequency } \\
\left(\mathrm{cm}^{-1}\right)\end{array}$ & Potential Energy Distribution ${ }^{a}$ \\
\hline 124 & $\mathrm{CNC}^{\alpha}(3) \operatorname{def}(26), \mathrm{NC}^{\alpha}(3) \mathrm{C} \operatorname{def}(10)$ \\
\hline 117 & $\mathrm{H}(5) \ldots \mathrm{O} \operatorname{str}(41), \mathrm{H}(2) \ldots \mathrm{O} \operatorname{str}(26)$ \\
\hline 116 & $\mathrm{H}(3) \cdots \mathrm{O} \operatorname{str}(33), \mathrm{H}(5) \cdots \mathrm{O} \operatorname{str}(31)$ \\
\hline 113 & $\mathrm{H}(1) \cdots \mathrm{O}(5) \operatorname{str}(33), \mathrm{H}(3) \cdots \mathrm{O} \operatorname{str}(23)$ \\
\hline 106 & $\mathrm{H}(1) \ldots \mathrm{O}(5) \operatorname{str}(28), \mathrm{NC}^{\alpha}(2) \operatorname{tor}(10)$ \\
\hline 85 & $\mathrm{H}(4) \ldots \mathrm{O}(2) \operatorname{str}(28), \mathrm{NH}(4)$ opb $(21)$ \\
\hline 80 & $\mathrm{NH}(1)$ opb $(10)$ \\
\hline 69 & $\mathrm{C}=\mathrm{O}(1) \ldots \mathrm{H} \operatorname{def}(47), \mathrm{C}^{\alpha}(1) \mathrm{C} \operatorname{tor}(11)$ \\
\hline 67 & $\mathrm{C}=\mathrm{O}(5) \ldots \mathrm{H}(1) \operatorname{def}(49), \mathrm{C}=\mathrm{O}(2) \ldots \mathrm{H}(4) \operatorname{def}(30)$ \\
\hline 65 & $\mathrm{C}=\mathrm{O}(4) \ldots \mathrm{H}$ def(50), $\mathrm{C}=\mathrm{O}(5) \ldots \mathrm{H}(1) \operatorname{def}(23)$ \\
\hline 63 & $\mathrm{H}(1) \ldots \mathrm{O}(5) \operatorname{str}(15), \mathrm{C}^{\beta}(1)$ bend $1(13)$ \\
\hline 61 & $\mathrm{C}^{\alpha}(4) \mathrm{C}$ tor $(17), \mathrm{NC}^{\alpha}(4)$ tor $(14)$ \\
\hline 54 & $\mathrm{NC}^{\alpha}(4)$ tor(12), $\mathrm{NH}(4)$ opb(11) \\
\hline 42 & $\mathrm{C}=\mathrm{O}(5) \ldots H(1) \operatorname{def}(20), \mathrm{C}^{\alpha}(4) \mathrm{C}$ tor(14) \\
\hline 36 & $\mathrm{NC}^{\alpha}(3)$ tor $(31), \mathrm{C}^{\alpha}(2) \mathrm{C}$ tor $(20)$ \\
\hline 35 & $\mathrm{NH}(4) \ldots \mathrm{O}(2) \operatorname{def}(41), \mathrm{NH}(1) \ldots \mathrm{O}(5) \operatorname{def}(11)$ \\
\hline 34 & $\mathrm{NH}(3) \ldots \mathrm{O} \operatorname{def}(45), \mathrm{NH}(2) \ldots \mathrm{O} \operatorname{def}(10)$ \\
\hline 34 & $\mathrm{NH}(5) \ldots \mathrm{O} \operatorname{def}(70), \mathrm{NH}(1) \ldots \mathrm{O}(5) \operatorname{def}(10)$ \\
\hline 28 & $\mathrm{NC}^{\mathrm{x}}(1)$ tor $(23), \mathrm{C}^{\mathrm{x} \times}(1) \mathrm{C}$ tor $(15)$ \\
\hline 27 & $\mathrm{C}^{\alpha}(4) \mathrm{C}$ tor $(25), \mathrm{NH}(4) \ldots \mathrm{O}(2) \operatorname{def}(15)$ \\
\hline 25 & $\mathrm{C}=\mathrm{O}(3) \operatorname{tor}(67)$ \\
\hline 24 & $\mathrm{C}=\mathrm{O}(2)$ tor $(53), \mathrm{C}=\mathrm{O}(4) \operatorname{tor}(11)$ \\
\hline 24 & $\mathrm{C}=\mathrm{O}(4)$ tor $(60), \mathrm{C}=\mathrm{O}(1)$ tor $(15)$ \\
\hline 21 & $\mathrm{NC}^{\alpha}(1)$ tor $(14), \mathrm{C}=\mathrm{O}(5)$ tor $(10)$ \\
\hline 17 & $\mathrm{NH}(4)$ tor $(61), \mathrm{NH}(5)$ tor $(11)$ \\
\hline 16 & $\mathrm{NH}(3) \operatorname{tor}(37), \mathrm{NH}(1)$ tor $(26)$ \\
\hline 16 & $\mathrm{NH}(2)$ tor $(63)$ \\
\hline 14 & $\mathrm{NC}^{\mathrm{a}}(3) \operatorname{tor}(19), \mathrm{NH}(4) \ldots \mathrm{O}(2) \operatorname{def}(12)$ \\
\hline
\end{tabular}

a Only contributions equal to or greater than $10 \%$ are included. Abbreviations used: asym, asymmetric; def, deformation; ipb, in-plane bend; opb, out-of-plane bend; str, stretch; sym, symmetric; tor, torsion.

structures $^{10,11}(0.37 \mathrm{D}$ for amide $\mathrm{I}$ and $0.27 \mathrm{D}$ for amide II) may not be appropriate for $\beta$-turns.

Although we have not included the detailed results for the calculations on $\beta$-turns of types $\mathbf{I}^{\prime}-\mathrm{III}^{\prime}$, the normal modes of these structure have been calculated. For purposes of comparison we present in Table $\mathrm{V}$ the amide I-III frequencies of these $\beta$-turns, the first two modes incorporating the effects of transition dipole coupling.

The complete effects of structural variations are difficult to present in a concise and easily digestible manner. In addition to the standard structures, we have computed the normal modes of seven different type I structures, seven different type II structures, and six different type III structures, and each would require a table such as Table I to list the predicted frequencies. It seems more useful at this stage to concentrate on the structural dependence of the characteristic amide I-III modes. We show these results in Fig. 2 for variations in $(\phi, \psi)_{2}$ and $(\phi, \psi)_{3}$ and in Fig. 3 for variations in $(\phi, \psi)_{1}$ and $(\phi, \psi)_{4}$. Since much information is contained in these figures, it is important to explain their content in detail. 
TABLE III

Calculated Frequencies of a Type III $\beta$-Turn of $\mathrm{CH}+\mathrm{CO}-(\mathrm{Ala})_{4}-\mathrm{NH}-\mathrm{CH}_{3}$

\begin{tabular}{|c|c|}
\hline $\begin{array}{l}\text { Frequency } \\
\qquad\left(\mathrm{cm}^{-1}\right)\end{array}$ & Potential Energy Distribution ${ }^{a}$ \\
\hline $3272-3269$ & NH str: 5 modes \\
\hline $2979-2977$ & $\mathrm{CH}_{3}$ asym str: 12 modes \\
\hline $2942-2941$ & $\mathrm{CH}_{3}$ sym str: 6 modes \\
\hline $2975-2874$ & $\mathrm{C}^{\prime \prime} \mathrm{H}^{*} \mathrm{str}: 4$ modes \\
\hline 1676 & $\mathrm{C}=\mathrm{O}(5) \operatorname{str}(68), \mathrm{CN}(5) \operatorname{str}(14)$ \\
\hline 1674 & $\mathrm{C}=\mathrm{O}(2) \operatorname{str}(58), \mathrm{C}=\mathrm{O}(1) \operatorname{str}(16), \mathrm{CN}(2) \operatorname{str}(10)$ \\
\hline 1671 & $\mathrm{C}=\mathrm{O}(1) \operatorname{str}(55), \mathrm{C}=\mathrm{O}(2) \operatorname{str}(17)$ \\
\hline 1667 & $\mathrm{C}=\mathrm{O}(3) \operatorname{str}(45), \mathrm{C}=\mathrm{O}(4) \operatorname{str}(32)$ \\
\hline 1665 & $\mathrm{C}=\mathrm{O}(4) \operatorname{str}(44), \mathrm{C}=\mathrm{O}(3) \operatorname{str}(32)$ \\
\hline 1578 & $\mathrm{NH}(5)$ ipb(32), $\mathrm{CN}(5) \operatorname{str}(17), \mathrm{C}^{\prime \prime} \mathrm{H}_{3}$ sym bend(28) \\
\hline 1563 & $\mathrm{NH}(1) \operatorname{ipb}(52), \mathrm{CN}(1) \operatorname{str}(27)$ \\
\hline 1550 & $\mathrm{NH}(2)$ ipb(38), CN(2) str(27), Cr(1)C $\operatorname{str}(15), \mathrm{C}=\mathrm{O}(2)$ ipb(12) \\
\hline 1543 & $\mathrm{NH}(4)$ ipb(48), $\mathrm{CN}(4) \operatorname{str}(32), \mathrm{C}=\mathrm{O}(4)$ ipb(11) \\
\hline 1536 & $\mathrm{NH}(3)$ ipb(44), CN(3) str(33), Cr(2)C $\operatorname{str}(11), \mathrm{C}=\mathrm{O}$ ipb(12) \\
\hline $1468-1451$ & $\mathrm{CH}_{3}$ asym bend: 12 modes \\
\hline $1380-1374$ & $\mathrm{CH}_{3}$ sym bend: 6 modes \\
\hline 1359 & $\mathrm{H}^{\prime \prime}(4)$ bend $1(45), \mathrm{NH}(5)$ ipb(14), $\mathrm{CH}_{3}(3)$ sym bend $(13)$ \\
\hline 1359 & $\mathrm{H}^{*}(3)$ bend $1(39), \mathrm{H}^{\star}(2)$ bend $1(16)$ \\
\hline 1357 & $\mathrm{H}^{\circ}(2)$ bend $1(43), \mathrm{H}^{\circ}(3)$ bend $1(21)$ \\
\hline 1351 & $H^{\prime \prime}(1)$ bend $1(41), H^{\prime \prime}(2)$ bend $1(19)$ \\
\hline 1321 & $\mathrm{NH}(1)$ ipb(30), $\mathrm{C}^{\prime} \mathrm{C} \operatorname{str}(17), \mathrm{CN}(1) \operatorname{str}(15), \mathrm{C}=\mathrm{O}(1) \mathrm{ipb}(13)$ \\
\hline 1317 & NH(4) ipb(20), C $\alpha(3) C \operatorname{str}(13)$ \\
\hline 1303 & $\mathrm{NH}(3)$ ipb(18), $\mathrm{C}^{\alpha}(2) \mathrm{C} \operatorname{str}(10)$ \\
\hline 1291 & $\mathrm{NH}(2)$ ipb(25), NH(3) ipb(14) \\
\hline 1286 & $\mathrm{NH}(5)$ ipb(35), $\mathrm{C}^{\sim}(4) \mathrm{C} \operatorname{str}(16), \mathrm{H}^{\sim}(4)$ bend $1(13), \mathrm{CN}(5) \operatorname{str}(12)$ \\
\hline 1212 & $N C^{a}(2) \operatorname{str}(30), N C^{a}(1) \operatorname{str}(22)$ \\
\hline 1194 & $\mathrm{NC}^{\prime \prime}(4) \operatorname{str}(22), \mathrm{NC}^{\prime \prime} \operatorname{str}(22)$ \\
\hline 1192 & $\mathrm{NC}^{\alpha}(4) \operatorname{str}(25), \mathrm{NC}^{\prime \prime} \operatorname{str}(21), \mathrm{CH}_{3}(2)$ rock $2(12)$ \\
\hline 1166 & $\mathrm{CN}^{\circ}(1) \operatorname{str}(25), \mathrm{NC}^{\circ}(2) \operatorname{str}(14), \mathrm{CH}_{3}(1) \operatorname{rock} 2(10)$ \\
\hline 1116 & $\mathrm{NC}^{\prime \prime} \operatorname{str}(18), \mathrm{CH}_{3}(3) \operatorname{rock} 2(14), \mathrm{C}^{\alpha}(4) \mathrm{C}^{\beta} \operatorname{str}(10)$ \\
\hline 1112 & $\mathrm{CH}_{3}(3)$ rock $1(17), \mathrm{C}^{\prime \prime} \mathrm{H}_{3}$ rock $2(17), \mathrm{C}^{\alpha}(4) \mathrm{C}^{\beta} \operatorname{str}(13)$ \\
\hline 1111 & $\mathrm{C}^{\prime} \mathrm{H}_{33}$ rock $1(32), \mathrm{C}^{\prime} \mathrm{H}_{3}$ rock $2(11), \mathrm{C}^{\natural}(1) \mathrm{C}^{3}$ str(10) \\
\hline 1106 & $\mathrm{CH}_{3}(3)$ rock $1(18), \mathrm{C}^{\alpha}(4) \mathrm{C}^{\mathrm{s}} \mathrm{str}(13)$ \\
\hline 1098 & $\mathrm{CH}_{3}(1)$ rock $1(17), \mathrm{CH}_{3}(1)$ rock $2(15)$ \\
\hline 1093 & $\begin{array}{l}\mathrm{C}^{\alpha}(3) \mathrm{C}^{\beta} \operatorname{str}(26), \mathrm{CH}_{3}(2) \text { rock } 2(23), \mathrm{C}^{\alpha}(2) \mathrm{C}^{3} \operatorname{str}(17), \mathrm{CH}_{3}(1) \text { rock } \\
\quad 1(12)\end{array}$ \\
\hline 1091 & $\begin{array}{l}\mathrm{C}^{\prime \prime}(2) \mathrm{C}^{\beta} \operatorname{str}(14), \mathrm{C}^{\alpha}(1) \mathrm{C}^{\beta} \operatorname{str}(11), \mathrm{C}^{\prime \prime}(3) \mathrm{C}^{\beta 3} \operatorname{str}(12), \mathrm{CH}_{3}(1) \text { rock } \\
\quad 1(10), \mathrm{CH}_{3}(2) \text { rock } 1(10)\end{array}$ \\
\hline 1084 & $\mathrm{C}^{\alpha}(2) \mathrm{C}^{\prime \prime} \operatorname{str}(15), \mathrm{C}^{\alpha}(1) \mathrm{C}^{\beta} \operatorname{str}(13), \mathrm{CH}_{3}(2)$ rock $2(11)$ \\
\hline 1078 & $\mathrm{C}^{\prime \prime} \mathrm{H}_{33}$ rock $1(55), \mathrm{C}^{\prime \prime} \mathrm{H}_{33}$ rock $2(22)$ \\
\hline 1068 & $\mathrm{C}^{\alpha}(3) \mathrm{C}^{\beta} \operatorname{str}(14), \mathrm{C}^{\alpha r}(2) \mathrm{C}^{\beta} \operatorname{str}(12)$ \\
\hline 1049 & $\mathrm{CH}_{3}(4)$ rock $1(56), \mathrm{CH}_{3}(4)$ rock $2(23)$ \\
\hline 1023 & $\mathrm{CH}_{3}(3)$ rock $1(30), \mathrm{NC}^{\prime \prime} \operatorname{str}(40), \mathrm{C}^{\prime \alpha}(4) \mathrm{C}^{\beta} \operatorname{str}(10)$ \\
\hline 1016 & $\mathrm{CH}_{3}(4)$ rock $2(43), \mathrm{CH}_{3}(4)$ rock $1(20)$ \\
\hline 995 & $\mathrm{H}^{\alpha(1)}$ bend $2(42)$ \\
\hline 992 & $\mathrm{H}^{\operatorname{er}(4)}$ bend $2(54), \mathrm{C}^{\alpha(}(4) \mathrm{C}^{\beta} \operatorname{str}(10)$ \\
\hline 984 & $H^{*}(2)$ bend $2(27), H^{\alpha}(3)$ bend $2(17), H^{\alpha}(1)$ bend $2(12)$ \\
\hline 981 & $\mathrm{H}^{\prime \prime}(3)$ bend $2(47), \mathrm{CH}_{3}(1)$ rock $2(10)$ \\
\hline
\end{tabular}




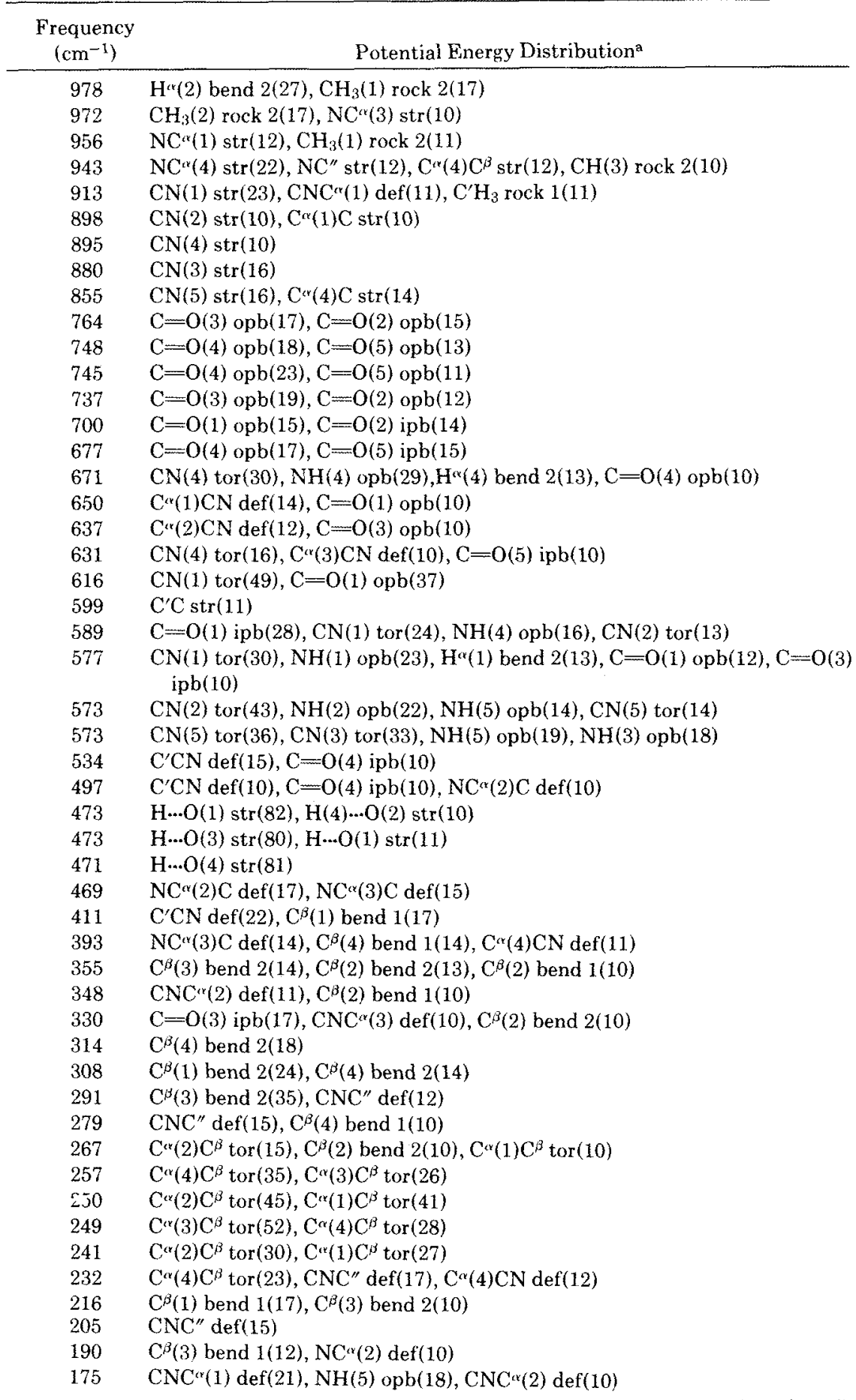




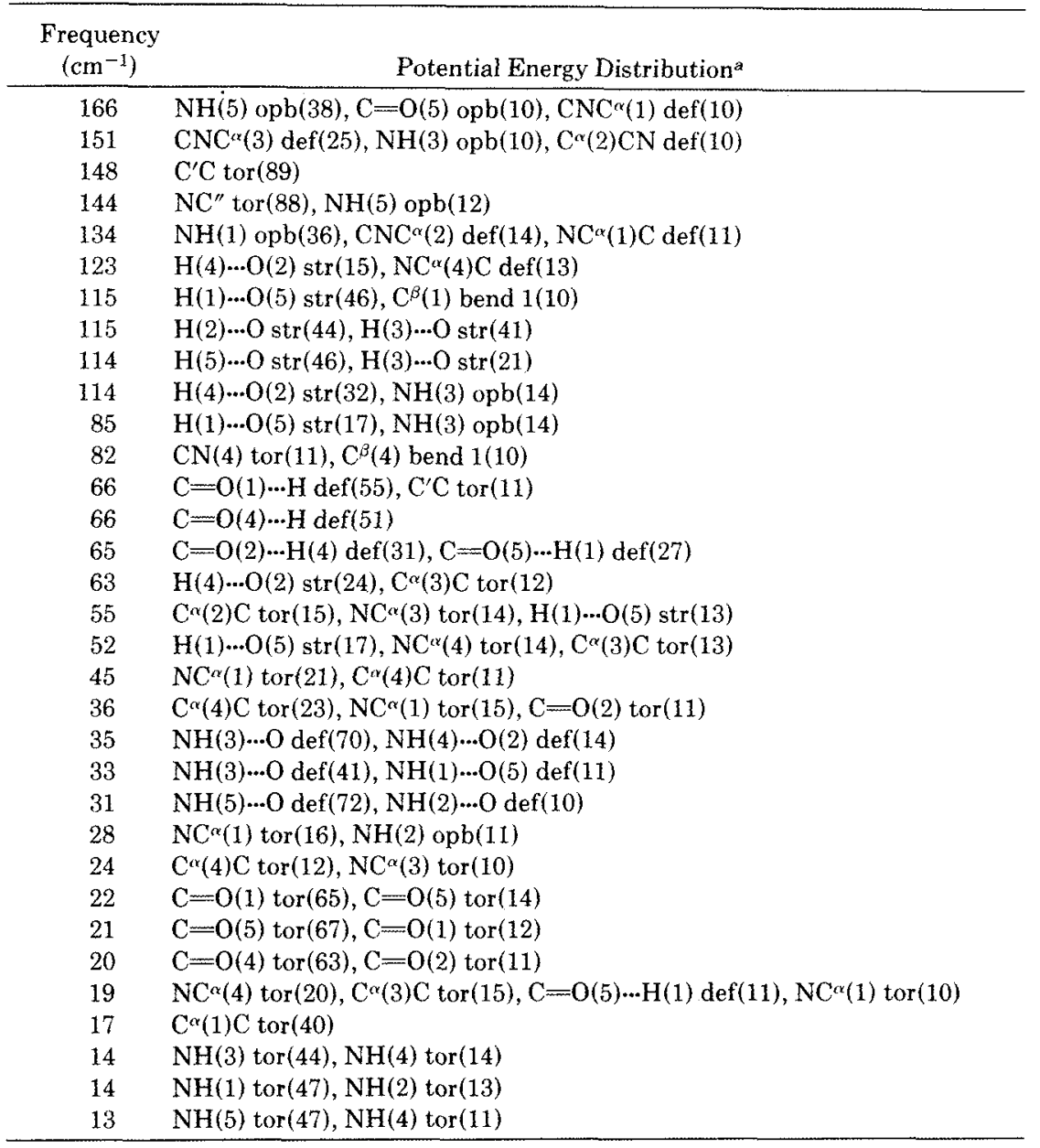

\footnotetext{
${ }^{a}$ Only contributions equal to or greater than $10 \%$ are included. Abbreviations used: asym, asymmetric; def, deformation; ipb, in-plane bend; opb, out-of-plane bend; str, stretch; sym, symmetric; tor, torsion.
}

Each figure consists of three parts, corresponding to the amide I-III frequencies. In each part the frequencies are given only for the amide modes of the peptide groups in the turn, viz., groups $2-4$ of Fig. 1. Each group is represented by a symbol, open if the mode is confined to that group and with an interior slash if it mixes with a similar mode in another group. If the second group is 2,3 , or 4 , it will have a similarly oriented slash; the absence of a second similar slash means that there is no significant mixing in the second mode or that the mixing is with other groups. Solid lines connect frequencies for type I $\beta$-turns, broken lines connect frequencies for type II $\beta$-turns, and dotted lines connect frequencies for type III $\beta$-turns (the connecting lines are for ease in reading and do not necessarily give the detailed variation of frequency with angle). In any particular graph only 


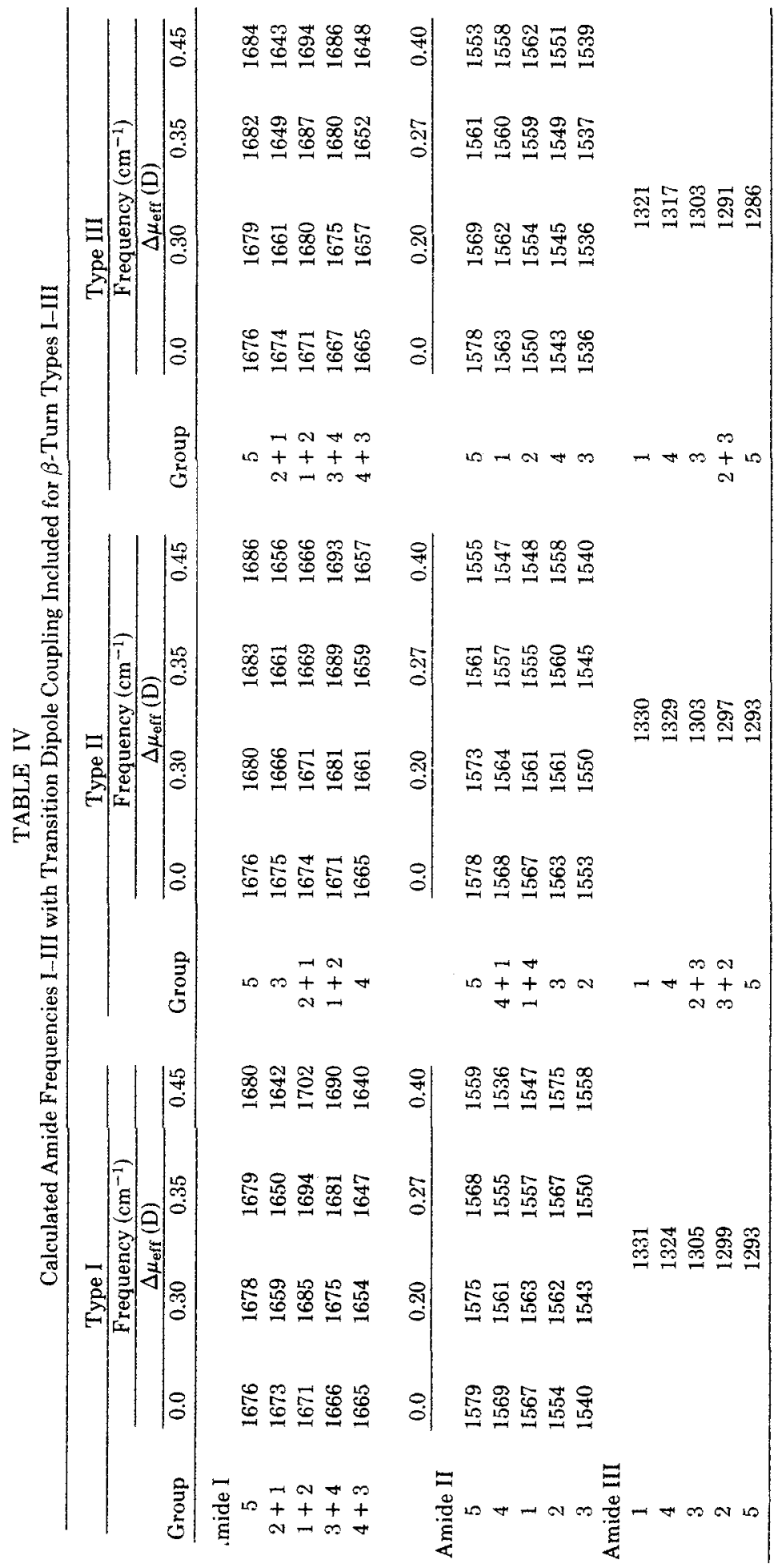




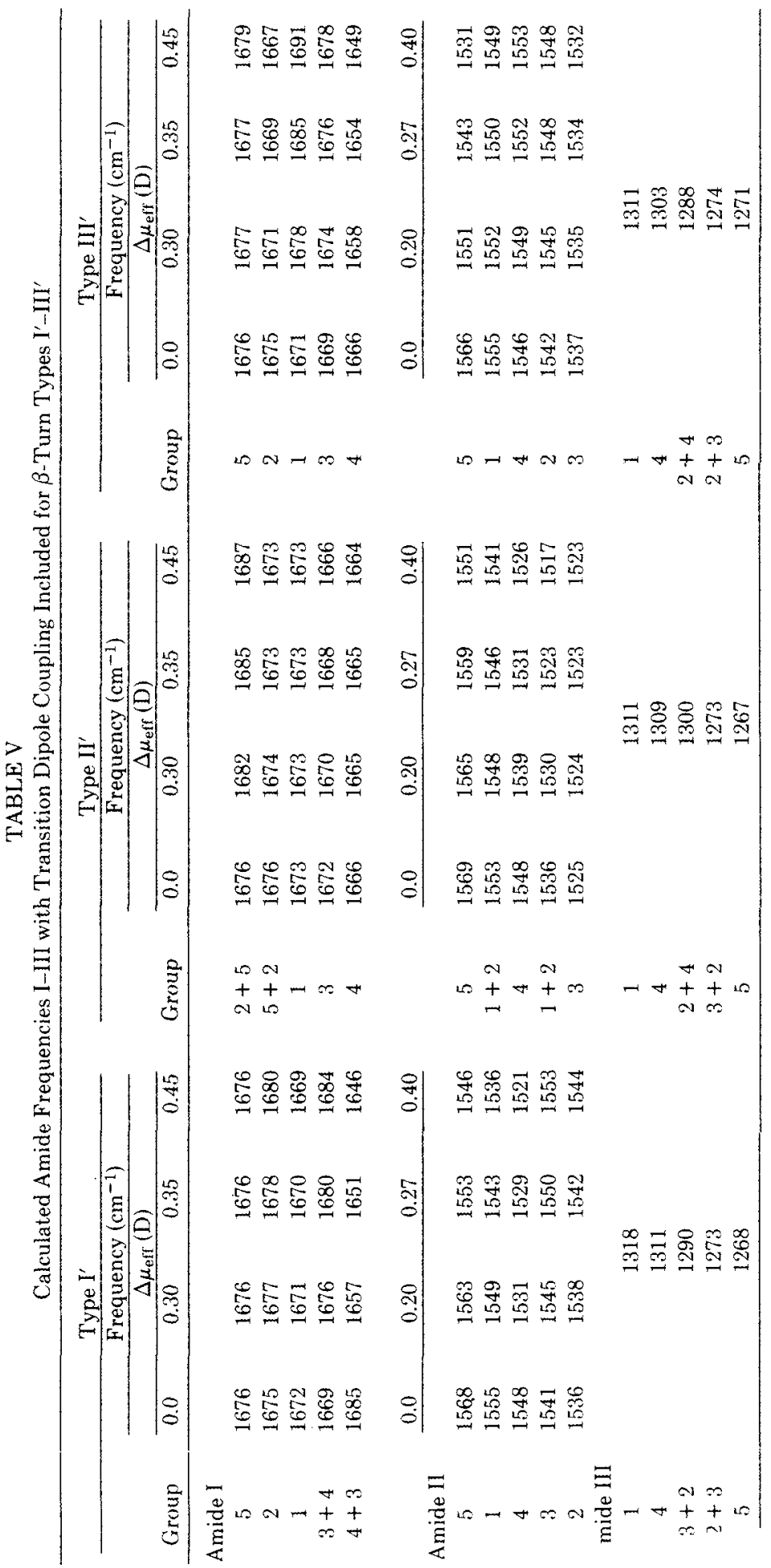



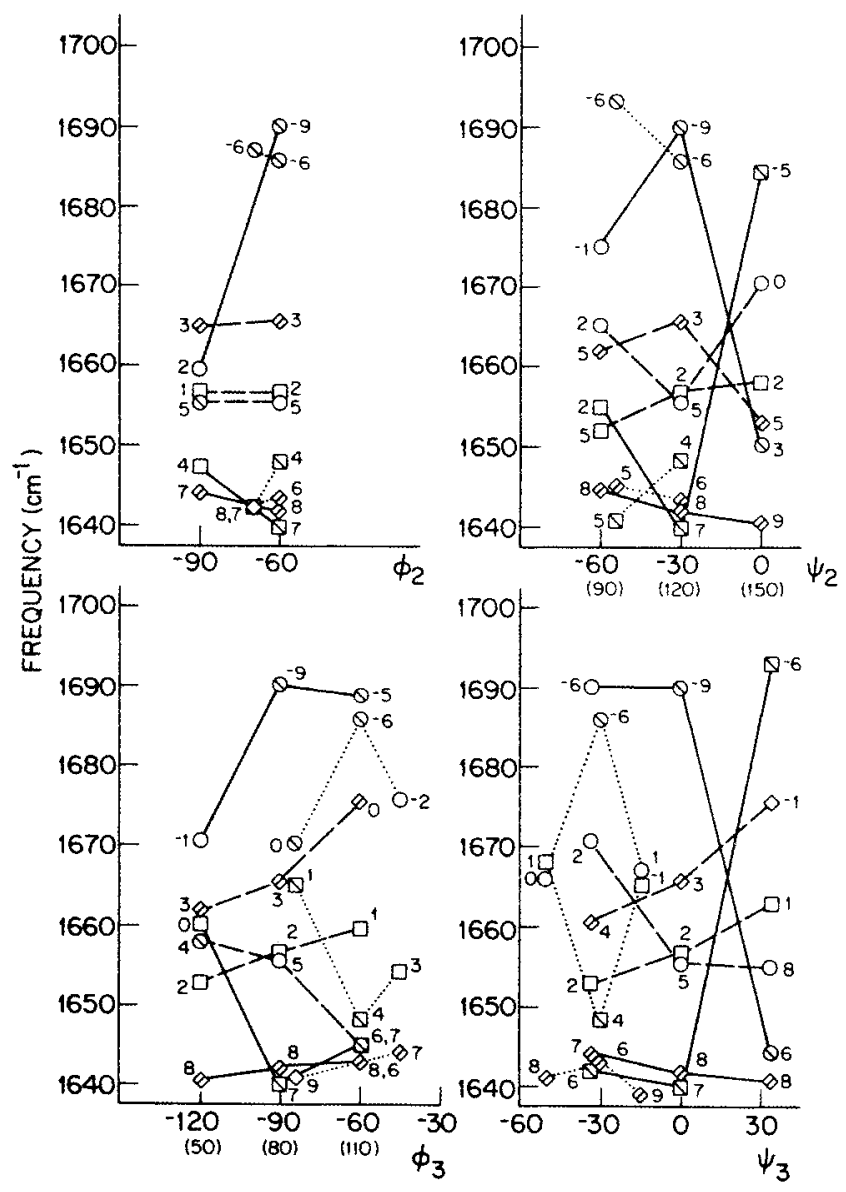

(a)

Fig. 2. Variation of frequency with $(\phi, \psi)_{2}$ and $(\phi, \psi)_{3}$ for (a) amide I, (b) amide II, and (c) amide III modes of types I ( --$)$, II (-- ) and III (..$) \beta$-turns. Symbols indicate peptide group: $\diamond, 2 ; 0,3 ; \square, 4$. See text for further details.

the denoted angle is varied; the other angles are kept constant at their standard values. The symbols represent the frequencies for the largest values of $\Delta \mu_{\text {eff }}$, viz., $0.45 \mathrm{D}$ for amide I and $0.40 \mathrm{D}$ for amide II. In order to provide some indication of the effect of change in $\Delta \mu_{\mathrm{eff}}$, we have placed a number next to each symbol to indicate the change in that frequency when $\Delta \mu_{\mathrm{eff}}$ is given the next lowest value that we used, viz., $0.35 \mathrm{D}$ for amide $\mathrm{I}$ and $0.27 \mathrm{D}$ for amide II. When two numbers refer to the same symbol (or symbols) it means that two frequencies overlap; in that case the first number refers to the mode of the lower-numbered $\beta$-turn (or lower-numbered peptide group).

One other structural variation was considered, viz., a type II $\beta$-turn 

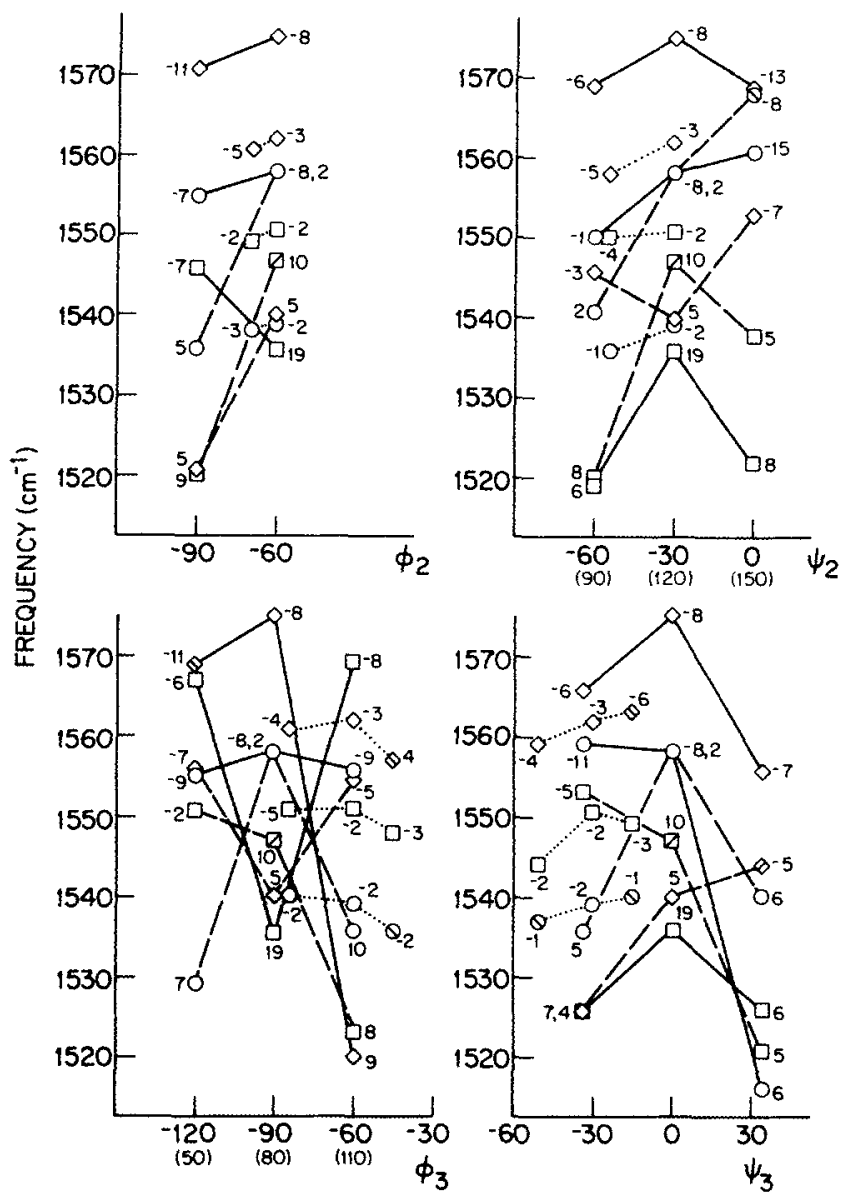

(b)

Fig. 2. (continued from the previous page)

without Gly in the third position, over a dozen of which are found in proteins. ${ }^{5}$ Of course, the standard dihedral angles are not stereochemicalfy feasible in this case. For this calculation we adopted values of $(\phi, \psi)_{2}=$ $-72^{\circ}, 119^{\circ}$ and $(\phi, \psi)_{3}=77^{\circ}, 16^{\circ}$, which represent the average values of these angles that we obtained from the Protein Data Bank structures. The normal modes were computed for a type II $\mathrm{CH}_{3}-\mathrm{CO}-(\mathrm{Ala})_{4}-\mathrm{NH}-\mathrm{CH}_{3}$ with the above angles and $(\phi, \psi)_{1}=(\phi, \psi)_{4}=-139^{\circ}, 135^{\circ}$. The amide I-III frequencies are given in Table VI as a function of $\Delta \mu_{\mathrm{eff}}$. 

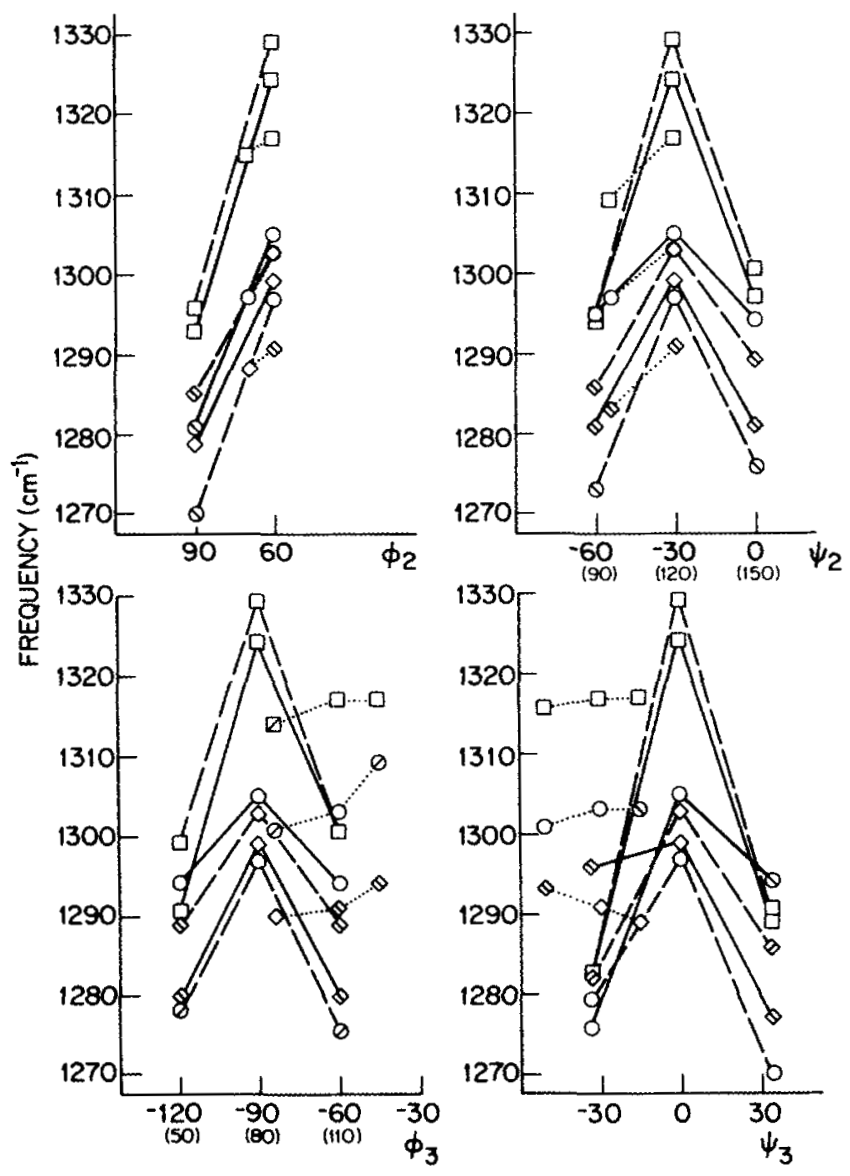

(c)

Fig. 2. (continued from the previous page)

\section{DISCUSSION}

\section{Standard $\beta$-Turns}

Several general features of the vibrational spectra of $\beta$-turns emerge from these calculations of the normal vibration frequencies. We will consider these here, deferring to later papers the specific assignments associated with particular $\beta$-turn structures. In what follows it should be kept in mind that the calculated frequencies cannot be expected to reproduce exactly the observed frequencies; our previous experience ${ }^{10-12}$ indicates that discrepancies of at least a few $\mathrm{cm}^{-1}$ would not be unusual. We discuss first the frequencies calculated for the characteristic amide modes, both for the $\beta$-turn structures as well as for the $\alpha$-helix and the antiparallel-chain 


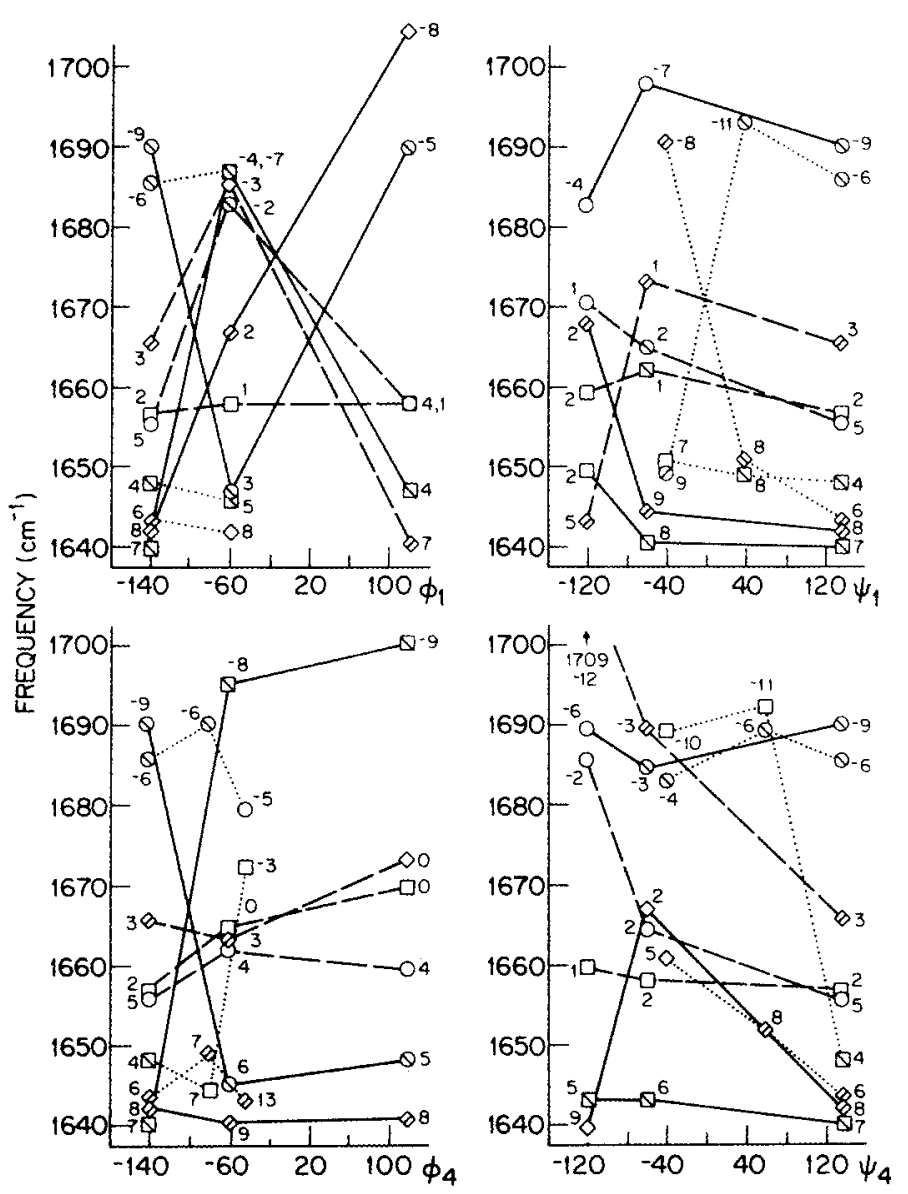

(a)

Fig. 3. Variation of frequency with $(\phi, \psi)_{1}$ and $(\phi, \psi)_{4}$ for (a) amide I, (b) amide II, and (c) amide III modes of types I ( $-\ldots)$, II ( $\cdots$, and III ( $\cdots) \beta$-turns. Symbols indicate peptide group: $\diamond, 2 ; 0,3 ; \square, 4$. See text for further details.

pleated sheet, $\beta(\mathbb{H})$. These results are collected in Table VII for groups $2-4$ in the turn.

With respect to the amide I modes, the frequencies associated with groups in the type II $\beta$-turn differ significantly from those predicted for types I and III. If we look only at the frequency ranges into which the modes fall for groups 2-4 (without regard to the group involved), then for the type I $\beta$-turn we expect frequencies near 1640 and $1690 \mathrm{~cm}^{-1}$; for the type II $\beta$-turn the comparable regions are about 1656 and $1666 \mathrm{~cm}^{-1}$; and for the type III $\beta$-turn these are $1646 \pm 3$ and $1686 \mathrm{~cm}^{-1}$. (These figures are applicable for $\Delta \mu_{\mathrm{eff}}=0.45 \mathrm{D}$. For $\Delta \mu_{\mathrm{eff}}=0.35 \mathrm{D}$ the numbers are somewhat different, but the same general observations are valid.) It might 

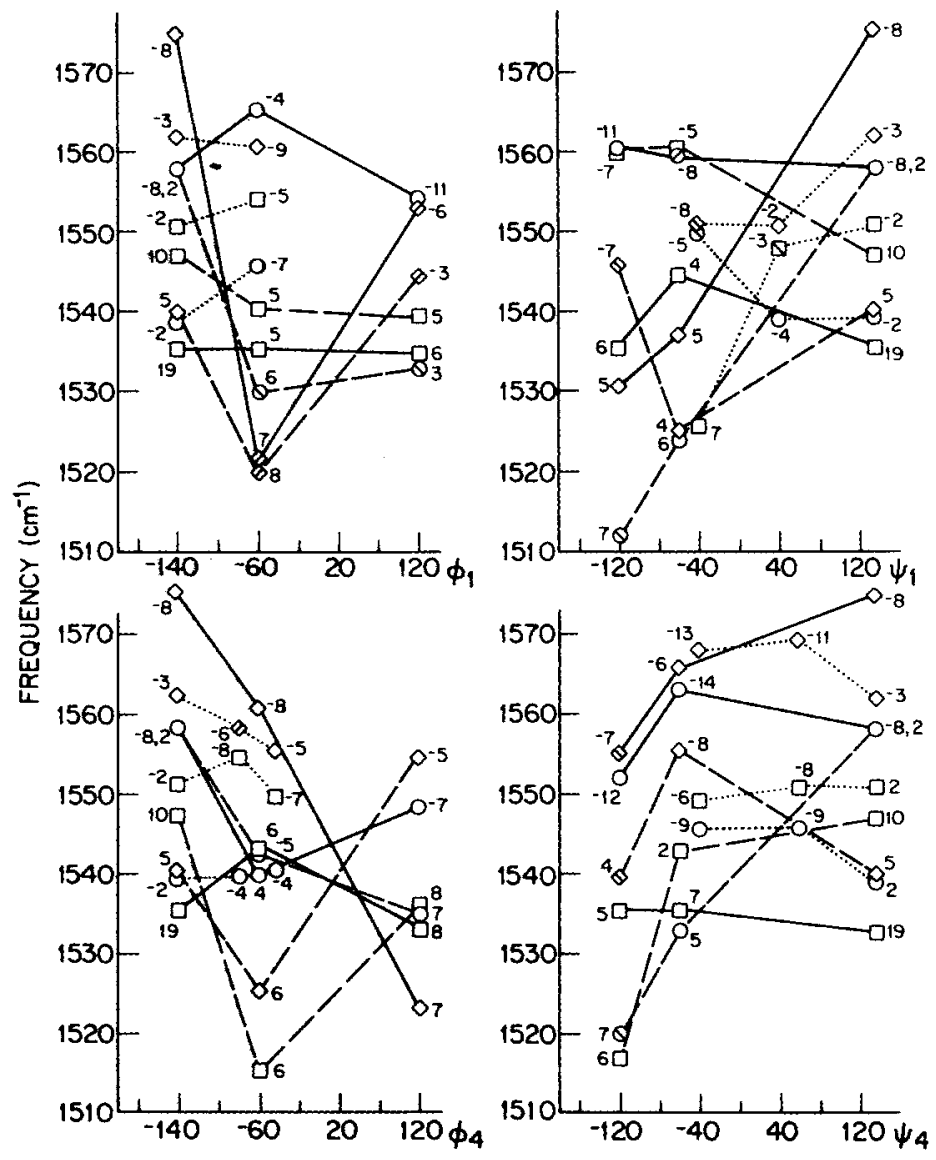

(b)

Fig. 3. (continued from the previous page)

be difficult to distinguish between types I and III (although the frequency gap between the ranges is larger for type I, viz., $50 \mathrm{~cm}^{-1}$, than for type III, viz., $\left.40 \mathrm{~cm}^{-1}\right)$, but the frequencies for type II and their gap $\left(10 \mathrm{~cm}^{-1}\right)$ are sufficiently distinct so that this $\beta$-turn should be clearly distinguishable from the others. (At this stage we leave out consideration of types I'-III', since they are found so much less frequently.)

In order to identify $\beta$-turns in the presence of other structures that occur in proteins and polypeptides, such as $\beta$-sheets and $\alpha$-helices, it is important to know whether the frequencies given above overlap those of the latter structures. For $\beta$-poly(L-alanine) our calculations ${ }^{11}$ predict the following amide I frequencies (which have observed counterparts): $1695 \mathrm{~cm}^{-1}$ (ir, $\|$ ), $1669 \mathrm{~cm}^{-1}$ (Raman), and $1630 \mathrm{~cm}^{-1}$ (ir, $\perp$ ). For $\alpha$-poly (L-alanine) our calculations $^{12}$ predict the following (observed) amide I frequency: 1659 

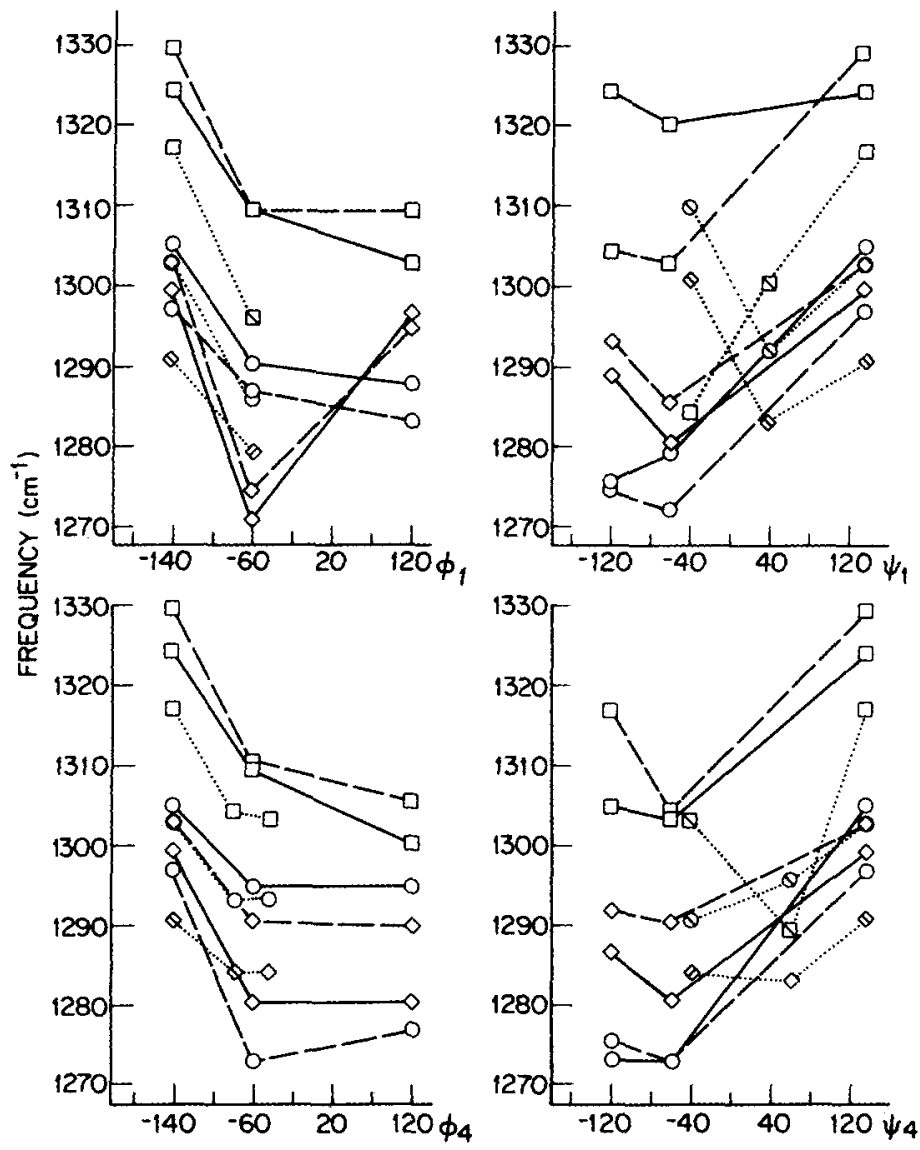

(c)

Fig. 3. (continued from the previous page)

$\mathrm{cm}^{-1}$ (Raman and ir, $\|$ ). Since $\beta$-turns lack symmetry, we would expect all of their normal modes to be potentially active in ir and Raman spectra. Allowing for the fact that observed bands for these structures cover a range of values, ${ }^{14}$ it seems reasonable to associate a range of $\pm 5 \mathrm{~cm}^{-1}$ with the $\beta$-sheet and $\alpha$-helix frequencies. Thus the $1640-\mathrm{cm}^{-1}$ band of the type I $\beta$-turn comes close to overlapping one band of the $\beta$-sheet, whereas the 1690 $\mathrm{cm}^{-1}$ band is essentially coincident with a $\beta$-sheet frequency. As we have already noted, ${ }^{1}$ this therefore means that the presence of a band near 1690 $\mathrm{cm}^{-1}$ in the ir spectrum of a protein should not be taken as a unique indication of the presence of antiparallel-chain pleated- $\beta$-sheet structure. For type II $\beta$-turns the predicted band at $1656 \mathrm{~cm}^{-1}$ overlaps the strong band of the $\alpha$-helix, whereas the $1666 \cdot \mathrm{cm}^{-1}$ band overlaps the Raman band of the $\beta$-sheet. Of course, in the latter case the $\beta$-turn would not exhibit ir 
TABLE VI

Calculated Frequencies with Transition Dipole Coupling Included for a Type II $\beta$-Turn of $\mathrm{CH}_{3}$ - CO-(Ala) $)_{4}-\mathrm{NH}-\mathrm{CH}_{3}$

\begin{tabular}{|c|c|c|c|c|}
\hline \multirow[b]{3}{*}{ Group } & \multicolumn{4}{|c|}{ Frequency $\left(\mathrm{cm}^{-1}\right)$} \\
\hline & \multicolumn{4}{|c|}{$\Delta \mu_{\mathrm{eff}}$} \\
\hline & 0.0 & 0.30 & 0.35 & 0.45 \\
\hline \multicolumn{5}{|l|}{ Amide I } \\
\hline $5+3$ & 1676 & 1676 & 1677 & 1679 \\
\hline $3+5$ & 1676 & 1665 & 1658 & 1653 \\
\hline $2+1$ & 1673 & 1672 & 1672 & 1671 \\
\hline $1+2$ & 1671 & 1685 & 1694 & 1702 \\
\hline \multirow[t]{2}{*}{4} & 1665 & 1662 & 1659 & 1659 \\
\hline & 0.0 & 0.20 & 0.27 & 0.40 \\
\hline \multicolumn{5}{|l|}{ Amide II } \\
\hline 5 & 1566 & 1563 & 1556 & 1551 \\
\hline $4+3$ & 1553 & 1548 & 1541 & 1536 \\
\hline 1 & 1551 & 1548 & 1540 & 1533 \\
\hline 4 & 1546 & 1541 & 1536 & 1531 \\
\hline 2 & 1534 & 1530 & 1527 & 1523 \\
\hline \multicolumn{5}{|c|}{ Amide III } \\
\hline 1 & & & & \\
\hline 4 & & & & \\
\hline $2+3$ & & & & \\
\hline $3+2$ & & & & \\
\hline 5 & & & & \\
\hline
\end{tabular}

bands near 1630 and $1690 \mathrm{~cm}^{-1}$. The frequencies of the type III $\beta$-turn would overlap less with those of the $\beta$-sheet than was the case for the type I $\beta$-turn, but the coincidences are close. Our calculations thus clearly indicate that caution is necessary in using the traditional frequency assignments of amide I bands in proteins, and especially in the interpretation of intensity changes in terms only of changes in $\alpha$-helix and $\beta$-sheet content. The situation is, of course, helped by obtaining both ir and Raman spectra, since, as can be seen from Table VII, various mutually exclusive assignments are possible.

The amide II modes of the $\beta$-turns seem to span fairly uniformly the range of about $1536-1562 \mathrm{~cm}^{-1}$, the type I $\beta$-turn being the only one with a distinctively high frequency of $1575 \mathrm{~cm}^{-1}$. The predicted (and observed) modes of the regular structures are somewhat lower: $1523-1555 \mathrm{~cm}^{-1}$ for the $\beta$-sheet ${ }^{11}$ and $1520-1543 \mathrm{~cm}^{-1}$ for the $\alpha$-helix. ${ }^{12}$ Therefore, it may be possible to infer the presence of type I $\beta$-turns from an analysis of the amide II region.

All of the amide III modes of the $\beta$-turn types I-III are predicted in the region of $1290-1330 \mathrm{~cm}^{-1}$, and these $\beta$-turns are probably not distinguishable from one another. What is interesting, however, is that this range is at a significantly higher frequency than those associated with $\beta$-sheet and $\alpha$-helix structures, thus providing a possibility for a diagnostic for $\beta$-turns. For $\beta$-poly(L-alanine) the calculated amide III modes occur in 
TABLE VII

Calculated Amide Mode Frequencies for Various Structures

\begin{tabular}{|c|c|c|c|c|c|}
\hline \multirow[b]{2}{*}{ Mode } & \multicolumn{5}{|c|}{ Structure } \\
\hline & $\alpha^{\mathrm{a}}$ & $\bar{\beta}(t)^{\mathrm{b}}$ & $\beta_{I}$ & $\beta_{\mathrm{II}}$ & $\beta_{\mathrm{HI}}$ \\
\hline \multirow[t]{5}{*}{ Amide I } & & {$[1701]^{\mathrm{c}}$} & & & \\
\hline & & 1695 & 1690 & & 1686 \\
\hline & [1663] & 1669 & & 1666 & \\
\hline & 1659 & & & $\sim 1656$ & \\
\hline & {$[1650]$} & 1630 & $\sim 1640$ & & $1646 \pm 3$ \\
\hline \multirow[t]{5}{*}{ Amide II } & & {$[1587]$} & 1575 & & \\
\hline & {$[1546]$} & 1555 & 1558 & 1558 & 1562 \\
\hline & 1543 & & & 1547 & 1551 \\
\hline & & 1534 & 1536 & 1540 & 1539 \\
\hline & 1520 & 1523 & & & \\
\hline \multirow[t]{7}{*}{ Amide III } & & & 1324 & 1329 & 1317 \\
\hline & & & 1305 & 1303 & 1303 \\
\hline & 1287 & & 1299 & 1297 & 1291 \\
\hline & 1270 & & & & \\
\hline & & 1247 & & & \\
\hline & & 1228 & & & \\
\hline & & 1225 & & & \\
\hline \multirow[t]{6}{*}{ Amide $\mathrm{V}$} & १39] & [709] & 595 & 644 & 671 \\
\hline & 028 & 713 & 575 & 607 & 589 \\
\hline & 609 & 702 & 574 & 594 & 577 \\
\hline & [559] & [699] & 570 & 588 & 573 \\
\hline & & & & 572 & 573 \\
\hline & & & & 571 & \\
\hline
\end{tabular}

a From Ref. 12.

b From Ref. 11.

c Not observed.

the 1225-1247- $\mathrm{cm}^{-1}$ region ${ }^{11}$ and are usually associated with strong Raman bands observed at frequencies no higher than $1240 \mathrm{~cm}^{-1} .14$ For $\alpha$-poly(L-alanine) the calculated modes are found in the $1270-1287-\mathrm{cm}^{-1}$ region ${ }^{12}$ but give rise to relatively weak ir and Raman bands (either because the NH ipb contribution is so low-about $10 \%$-or because $\mathrm{CN}$ str makes very little contribution). It is therefore possible that bands associated with $\beta$-turns can be observed in the region near and above $1300 \mathrm{~cm}^{-1}$.

Of course, it must be remembered that the amide III mode is sensitive to side-chain composition and to backbone conformation, ${ }^{14}$ and it therefore must be used with caution in characterizing peptide structure. In the above examples of poly(L-alanine), this mode has significantly different character in the two structures. In $\beta$-poly (L-alanine), bands are observed at $\sim 1222$ and $1242 \mathrm{~cm}^{-1}$, which have the usual amide III character: $\mathrm{NH}$ ipb(17) + $\mathrm{CN}$ str(14) in the first case and $\mathrm{NH}$ ipb(19) + CN str(18) in the second. The observed Raman band at $1368 \mathrm{~cm}^{-1}$ is mainly $\mathrm{H}^{\alpha}$ bend(22), $\mathrm{C}^{\alpha} \mathrm{C}$ str(17), and $\mathrm{CH}_{3}$ sym bend(13), but it also includes an $\mathrm{NH}$ ipb(20) contribution. On the other hand, in $\alpha$-poly (L-alanine) no band in this region exhibits the typical amide III character, viz., $\mathrm{NH}$ ipb + CN str: $\mathrm{CN}$ str does 
not appear at the $10 \%$, or above, level, and NH ipb contributes at this level to five bands in the $1270-1346-\mathrm{cm}^{-1}$ region. The situation for the $\beta$-turns exhibits similar differences (cf. Tables I-III). For the type I $\beta$-turn the frequencies associated with the turn $\left(1324,1305\right.$, and $\left.1299 \mathrm{~cm}^{-1}\right)$ all involve $\mathrm{NH}$ ipb $+\mathrm{CN}$ str. For the type II $\beta$-turn, $\mathrm{CN}$ str contributes to none of the three modes, which involve only NH ipb (and some CC str), although $\mathrm{NH}(2)$ ipb and $\mathrm{NH}(3)$ ipb mix for two of these. For the type III $\beta$-turn there is again no $\mathrm{CN}$ str contribution, and only one mode shows a significant admixture of two NH ipb contributions. A study of actual structures will be necessary to reveal the characteristic intensities associated with these modes (and, in fact, whether transition dipole coupling must be included to explain amide III frequencies).

The amide $\mathrm{V}$ modes, associated with $\mathrm{NH}$ opb coordinates, have been shown ${ }^{15}$ to be particularly sensitive to conformation: they are assigned to bands in the $610-620 \mathrm{~cm}^{-1}$ region for $\alpha$-helical structures and in the $700-705-\mathrm{cm}^{-1}$ region for $\beta$-sheet structures. ${ }^{16}$ These assignments are supported by detailed normal vibration calculations, ${ }^{11,12}$ which also show that other internal coordinates make varying contributions to the bands observed for these structures. What is particularly interesting is that although the frequency regions associated with the $\beta$-turns overlap, they are in general lower than the observed bands for the $\alpha$-helix: $570-575$ and 595 $\mathrm{cm}^{-1}$ for type I, 571-607 and $644 \mathrm{~cm}^{-1}$ for type II, and 573-589 and 671 $\mathrm{cm}^{-1}$ for type III (see Table VII). The highest frequency for each type of $\beta$-turn is distinctive for that turn, and may be a diagnostic for the structure. Of course, these frequency predictions say nothing regarding relative intensities expected for observed bands; these will have to await an analysis of actual structures. Nevertheless, our calculations suggest that the amide $V$ modes may be useful in identifying the presence of $\beta$-turns, and perhaps even in distinguishing between various types.

The amide VI modes, associated with $\mathrm{C}=\mathrm{O}$ opb coordinates, were originally ${ }^{15}$ thought to be characteristically observed near $600 \mathrm{~cm}^{-1}$, but detailed calculations indicate that they are very sensitive to side-chain structure and main-chain conformation. In polyglycine $I^{10}$ the main amide VI modes are calculated in the frequency range of $585-618 \mathrm{~cm}^{-1}$, with corresponding bands being observed at 589 (ir and Raman) and 614 (ir) $\mathrm{cm}^{-1}$. In $\beta$-poly (L-alanine) ${ }^{11}$ these modes shift up to the $682-703-\mathrm{cm}^{-1}$ region, the only assignable band being a Raman band at $698 \mathrm{~cm}^{-1}$. And in $\alpha$-poly(L-alanine) ${ }^{12}$ the main amide VI modes are calculated in the region of 701-782 $\mathrm{cm}^{-1}$, with observed bands being found at 692 (ir and Raman), 756 (Raman), and 774 (ir and Raman) $\mathrm{cm}^{-1}$. Thus in known regular conformations, amide VI modes span a range of almost $200 \mathrm{~cm}^{-1}$. For the $\beta$-turns we calculate the following ranges for the main amide VI modes: type I, 721-762 $\mathrm{cm}^{-1}$; type II, 724-747 $\mathrm{cm}^{-1}$; type III, 737-764 $\mathrm{cm}^{-1}$. Since these ranges significantly overlap that of the $\alpha$-helix (and for a type I $\beta$-turn possibly that of the $\beta$-sheet), and since amide VI modes (in distinction to amide $\mathrm{V}$ ) tend to be weak bands, it seems that these modes will be less 
useful in identifying $\beta$-turns than those listed in Table VII. Again, however, more definitive assessments must await detailed experimental studies of $\beta$-turn structures.

We have made only a cursory examination of the low-frequency region for the $\beta$-turn structures, even though this region might be expected to be quite sensitive to backbone conformation. The various modes in this region have frequencies that are generally within about $10 \mathrm{~cm}^{-1}$ of one another, so that a distinction between different types of $\beta$-turns does not seem to be feasible. The composition of these modes does, however, differ from one $\beta$-turn to another, and this may give rise to characteristic intensity differences between different structures. Again, a detailed understanding will probably have to await a study of some known structures.

\section{Structural Variations}

The most general statement that can be made about the effects of variations in structure of $\beta$-turns on their characteristic amide frequencies is that changes in conformation cause these frequencies to shift, usually by significant amounts. 'The origins of these shifts differ for the different modes; we will consider first the reasons for this.

In the case of amide I, the shifts in frequency with structure arise primarily from the effects of variation in transition dipole coupling. As can be seen by comparing Tables I-III, the amide I frequencies of $\beta$-turn types I-III are essentially the same in the absence of transition dipole coupling. Nor are these frequencies affected much by varying the $\phi, \psi$ over the range we have considered (although it should be noted that the mixing between different group modes does change with variation in the torsion angles). It is only when transition dipole coupling is included, with its explicit dependence on the separation and relative orientation of the $\mathrm{C}=\mathrm{O}$ stretching transition moments, 8,9 that large frequency splittings result which vary sensitively with the structural parameters. This is, of course, a consequence of the significant coupling that is introduced between amide I vibrations of adjacent peptide groups. As can be seen from Figs. 2(a) and 3(a), although some modes of the peptide groups in the turn are influenced more than others by a change in a given torsion angle, changes in each of the torsion angles $(\phi, \psi)_{1}$ to $(\phi, \psi)_{4}$ affect some of the frequencies. In particular, this is true of $\phi_{2}$ and $\psi_{3}$, so that it is not possible to attribute shifts in characteristic amide frequencies to variations in local conformational parameters alone, viz., $\psi_{2}$ and $\phi_{3}$ in this case. Nonadjacent torsion angles have a significant influence on the amide I frequencies of a given peptide group.

For the amide II modes the frequency dependence on conformation arises from coupling effects due to the internal force field as well as from transition dipole coupling. As can be seen from Tables I-III, even in the absence of transition dipole coupling, the amide II frequencies of peptide groups 3 and 
4 vary significantly between $\beta$-turn types I-III: for group 3 the frequencies are similar for types I and III but significantly different for type II, and for group 4 the frequencies are similar for types I and II but significantly different for type III. An analogous variation occurs when the torsion angles are changed, the frequencies of group 2 also being affected in this case. Of course, when transition dipole coupling is included, additional frequency shifts occur, the total changes with angle being given in Figs. 2(b) and 3(b). Again we see that amide II frequencies are influenced significantly by the values of nonadjacent torsion angles.

The amide III modes are not perturbed significantly by transition dipole coupling, and the dependence of frequency on conformation in this case arises entirely from internal coupling effects and changes in $\mathrm{H}_{4} \cdots \mathrm{O}_{2}$ and $\mathrm{H}_{1} \ldots \mathrm{O}_{5}$ hydrogen bond distances. As can be seen from Figs. 2(c) and 3(c), nonadjacent torsion angles are again influential in affecting the amide III frequency. Incidentally, since all torsion angles have a comparable effect on the amide III frequency, it is not possible to correlate this frequency with $\psi_{2}$ alone, as has been suggested. ${ }^{17}$ In fact, as we have pointed out, ${ }^{1}$ such a correlation would place $\beta$-turn frequencies near $1275 \mathrm{~cm}^{-1}$ for types I and III and near $1230 \mathrm{~cm}^{-1}$ for type II, whereas our calculations predict these frequencies to be in the $1290-1330-\mathrm{cm}^{-1}$ region (cf. Table IV).

The specific effects of changes in $\phi, \psi$ on the frequencies of $\beta$-turn vibrations, depicted in Figs. 2 and 3, are clearly quite complex. Some angular changes produce large, and others small, frequency shifts, and these vary with the type of $\beta$-turn. The changes shown in Figs. 2 and 3 are due to single angle changes; the effect of altering two angles simultaneously has not yet been explored in detail. At this stage it is, therefore, probably more useful to use these results only as a general indication of how conformational changes can influence the frequencies, leaving specific predictions to the cases of actual structures.

For the case of a type II $\beta$-turn with alanine in the third position, whose $(\phi, \psi)$ differ slightly from those of the standard type II turn, it is interesting to observe that significant frequency shifts occur. In the first place, it will be seen (cf. Tables IV and VI) that the amide II and III modes of the alanyl structure are predicted at frequencies of $\sim 15-25 \mathrm{~cm}^{-1}$ lower than those of the glycyl structure, despite the fact that the amide I frequencies are within a few $\mathrm{cm}^{-1}$ of each other. Moreover, the values of the amide II and III frequencies are close to those of the $\alpha$-helix, thus indicating that overlap for these structures is highly probable in these regions. The amide $\mathrm{V}$ modes of the alanyl type $I I \beta$-turn are predicted at $648,602,587,572$, and $570 \mathrm{~cm}^{-1}$, frequencies which are not significantly different from those of the glycyl type II $\beta$-turn. It would thus seem that the alanyl type II $\beta$-turn is distinguishable from other $\beta$-turns by its low amide II and III frequencies, but it may be difficult to distinguish from the $\alpha$-helix unless its amide $V$ mode shows up at a lower frequency. 


\section{CONCLUSIONS}

The calculations of the normal vibrations of $\beta$-turns provide important insights into the characteristic frequencies of these structures. In particular, they show that some of the modes have frequencies that overlap with those of the main regular structures, viz., the $\alpha$-helix and the $\beta(+t)$-sheet. To the extent that this occurs, it is clear that a more sophisticated interpretation of the vibrational spectra of proteins will be necessary if reliable structural assignments are to be made. On the other hand, some $\beta$-turn frequencies are characteristic of these structures, thus providing the possibility of identifying their presence from the ir and Raman spectra.

Another important conclusion of the present work is that the amide III modes of $\beta$-turns generally occur at frequencies significantly higher (viz., $\sim 1300-1330 \mathrm{~cm}^{-1}$ ) than those of $\alpha$-helix and $\beta$-sheet structures. This prediction is supported by recent studies $^{18}$ on a type $\mathrm{I} \beta$-turn tetrapeptide of known structure, in which a strong ir band observed at $1294 \mathrm{~cm}^{-1}$ (and calculated at $1291 \mathrm{~cm}^{-1}$ ) is found to disappear on deuteration. It should be noted that the general frequency region in which the amide III modes are expected will depend sensitively on the nature of the external and internal hydrogen bonding. We have already $\operatorname{seen}^{1}$ that in the absence of external hydrogen bonds, the amide III frequencies are $\sim 20 \mathrm{~cm}^{-1}$ lower. The present structure variation studies show that when internal hydrogen bonds are weakened (which occurs since the $\mathrm{H}$... O distances are increased by most changes in $\phi$ or $\psi$ ), the amide III frequencies are also decreased. It is thus possible that the lower-frequency bands $\left(1266-1278 \mathrm{~cm}^{-1}\right) \mathrm{re}$ cently found in the Raman spectra of oxytocin and its analogs ${ }^{19}$ and related hormones, ${ }^{20}$ and which have been assigned to amide III modes of postulated $\beta$-turns in these molecules, are a result of weaker hydrogen bonds associated with these conformations.

In subsequent papers on this subject we will deal with assignments for $\beta$-turns in specific structures. Because the $\phi, \psi$ in these may be slightly different from the "standard" values, their frequencies may vary somewhat from those presented here. The present calculations, however, provide a basis for general assignments of $\beta$-turn structures in peptides and proteins in that they indicate the key frequency regions to be looked at.

This research was supported by National Science Foundation Grants PCM76-83047 and CHE78-00753. One of us (J.B.) wishes to acknowledge support from a postdoctoral fellowship from the Macromolecular Research Center.

\section{References}

1. Bandekar, J. \& Krimm, S. (1979) Proc. Natl. Acad. Sci. USA 76, 774-777.

2. Venkatachalam, C. M. (1968) Biopolymers 6, 1425-1436.

3. Geddes, A. J., Parker, K. D., Atkins, E. D. T. \& Beighton, E. (1968) J. Mol. Biol. 32, $343-358$.

4. Lewis, P. N., Momany, F. A. \& Scheraga, H. A. (1973) Biochim. Biophys. Acta 303, 211-229.

5. Chou, P. Y. \& Fasman, G. D. (1977) J. Mol. Biol. 115, 135-175. 
6. Shields, J. E., McDowell, S. T., Pavlos, J. \& Gray, G. R. (1968) J. Am. Chem. Soc. 90, $3549-3556$.

7. Kawai, M. \& Fasman, G. (1978) J. Am. Chem. Soc. 100, 3630-3632.

8. Krimm, S. \& Abe, Y. (1972) Proc. Nat. Acad. Sci. USA 69, 2788-2792.

9. Moore, W. H. \& Krimm, S. (1975) Proc. Natl. Acad. Sci. USA 72, 4933-4935.

10. Moore, W. H. \& Krimm, S. (1976) Biopolymers 15, 2439-2464.

11. Moore, W. H. \& Krimm, S. (1976) Biopolymers 15, 2465-2483.

12. Rabolt J. F., Moore, W. H. \& Krimm, S. (1977) Macromolecules 10, 1065-1074.

13. Moore, W. H. \& Krimm, S. (1973) Spectrochim. Acta, Part A 29, 2025-2042.

14. Hsu, S. L., Moore, W. H. \& Krimm, S. (1976) Biopolymers 15, 1513-1528.

15. Miyazawa, T. (1967) Poly- $\alpha$-Amino Acids, Fasman, G. D., Ed., Dekker, New York, p. 69.

16 Masuda, Y., Fukushima, K., Fujii, T. \& Miyazawa, T. (1969) Biopolymers 8, 91-99.

17. Lord R. C. (1977) Appl. Spectrosc. 31, 187-194.

18. Bandekar, J. \& Krimm, S. (1979) Sixth American Peptide Symposium, Washington, D.C., Paper C-37.

19. Hruby, V. J., Deb, K. K., Fox, J., Bjarnason, J. \& Tu, A. T. (1978) J. Biol. Chem. 253, 6060-6067.

20. Tu, A. T., Lee, J., Deb, K. K. \& Hruby, V. J. (1979) J. Biol. Chem. 254, 3272-3278.

Received April 6, 1979

Accepted August 6, 1979 